NSC-NCTS-011026

FERMILAB-Pub-01/333-T

\title{
Kaluza-Klein States of the Standard Model Gauge Bosons: Constraints From High Energy Experiments
}

\author{
Kingman Cheung ${ }^{a}$ and Greg Landsberg ${ }^{b *}$ \\ a National Center for Theoretical Science, \\ National Tsing Hua University, Hsinchu, Taiwan ${ }^{\dagger}$ \\ ${ }^{b}$ Department of Physics, Brown University, Providence, RI 02912, USA
}

(Dated: October 25, 2018)

\begin{abstract}
In theories with the standard model gauge bosons propagating in $\mathrm{TeV}^{-1}$-size extra dimensions, their Kaluza-Klein states interact with the rest of the SM particles confined to the 3-brane. We look for possible signals for this interaction in the present high-energy collider data, and estimate the sensitivity offered by the next generation of collider experiments. Based on the present data from the LEP 2, Tevatron, and HERA experiments, we set a lower limit on the extra dimension compactification scale $M_{C}>6.8 \mathrm{TeV}$ at the $95 \%$ confidence level (dominated by the LEP 2 results) and quote expected sensitivities in the Tevatron Run 2 and at the LHC.
\end{abstract}

PACS numbers: $04.50 .+\mathrm{h}, 11.10 . \mathrm{Kk}, 11.25 . \mathrm{Mj}, 04.80 . \mathrm{Cc}$

\section{INTRODUCTION}

A possibility that the universe has additional compactified spatial dimensions beyond the familiar four-dimensional space-time has been long discussed [1]. Advances in modern string theory, along with the continuous attempts to solve the hierarchy problem of the standard model (SM), have revived interests to this subject. Recently, it has been suggested that the Planck, string, and grand unification scales can all be significantly lower than it was previously thought, perhaps as low as a few $\mathrm{TeV}$ [2]. For example, in a viable model suggested by Arkani-Hamed, Dimopoulos, and Dvali [3], the matter is confined to a 3-brane while gravity propagates in extra dimensions of a sub-millimeter size. In this model, the

\footnotetext{
* E-mail addresses: cheung@phys.cts.nthu.edu.tw and landsberg@hep.brown.edu

$\dagger$ The initial stage of the work was performed at the Davis Institute for High Energy Physics at the University of California, Davis, CA 95616, U.S.A.
} 
effective Planck scale is as low as a TeV, thus eliminating the hierarchy problem of the SM. This also yields rich phenomenology within the reach of future collider experiments, including

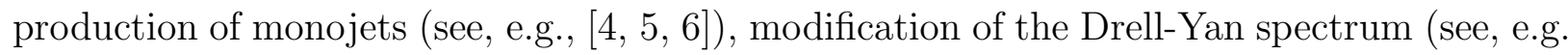
[4, 6, []), and even creation of mini black-holes and string-balls [8]. (For a brief summary of current experimental situation, see Ref. [9].)

A more generic picture drawn in string theories is that the SM matter particles reside on a $p$-brane $(p=\delta+3$; the space-time dimension of the brane is then $p+1)$ while gravity propagates in the entire ten-dimensional bulk. The compactification of the $\delta$ dimensions occurs internally within the brane, while the remaining $(6-\delta)$ dimensions are compactified transverse to the brane. Various phenomenology arises, depending on the relative magnitude of the two compactification scales, the string scale, and the Planck scale. The model of Arkani-Hamed, Dimopoulos, and Dvali [3] is a specific example with $\delta=0$.

Another interesting model was also proposed [10, 11], in which matter resides on a $p$ brane ( $p>3)$, with chiral fermions confined to the ordinary three-dimensional world internal to the $p$-brane and the SM gauge bosons also propagating in the extra $\delta>0$ dimensions internal to the $p$-brane. (Gravity in the bulk is not of direct concern in this model.) It was shown [10] that in this scenario it is possible to achieve the gauge coupling unification at a scale much lower than the usual GUT scale, due to a much faster power-law running of the couplings at the scales above the compactification scale of the extra dimensions. The SM gauge bosons that propagate in the extra dimensions compactified on $S^{1} / Z_{2}$, in the fourdimensional point of view, are equivalent to towers of Kaluza-Klein (KK) states with masses $M_{n}=\sqrt{M_{0}^{2}+n^{2} / R^{2}}(n=1,2, \ldots)$, where $R=M_{C}^{-1}$ is the size of the compact dimension, $M_{C}$ is the corresponding compactification scale, and $M_{0}$ is the mass of the corresponding SM gauge boson.

There are two important consequences of the existence of the KK states of the gauge bosons in collider phenomenology. (i) Since the entire tower of KK states have the same quantum numbers as their zeroth-state gauge boson, this gives rise to mixings among the zeroth (the SM gauge boson) and the $n$ th-modes $(n=1,2,3, \ldots)$ of the $W$ and $Z$ bosons. (The zero mass of the photon is protected by the $\mathrm{U}(1)_{\mathrm{EM}}$ symmetry of the SM.) (ii) In addition to direct production and virtual exchanges of the zeroth-state gauge bosons, both direct production and virtual effects of the KK states of the $W, Z, \gamma$, and $g$ bosons would become possible at high energies. 
In this paper, we study the effects of virtual exchanges of the KK states of the $W, Z, \gamma$, and $g$ bosons in high energy collider processes. While the effects on the low-energy precision measurements have been studied in detail [12, 13, 14, 15, 16, 17, 18] (we shall briefly summarize their findings in a later section), their high-energy counterparts have not been systematically studied yet. We attempt to bridge this gap by analyzing all the available highenergy collider data including the dilepton, dijet, and top-pair production at the Tevatron; neutral and charged-current deep-inelastic scattering at HERA; and the precision observables in leptonic and hadronic production at LEP 2.

We fit the observables in the above processes to the sum of the SM prediction and the contribution from the KK states of the SM gauge bosons. In all cases, the data do not require the presence of the KK excitations, which is then translated to the limits on the compactification scale $M_{C}$. The fit to the combined data set yields a $95 \%$ C.L. lower limit on $M_{C}$ of $6.8 \mathrm{TeV}$, which is substantially higher than that obtained using only electroweak precision measurements. In addition, we also estimate the expected reach on $M_{C}$ in Run 2 of the Fermilab Tevatron and at the LHC, using dilepton production.

The organization of this paper is as follows: In the next section, we describe the Lagrangian for the model [11], which has one extra dimension. In Sec. [1] we briefly summarize the existing constraints from precision measurements. In Sec. [V], we briefly discuss the effects of the KK states of the $Z$ boson on the atomic parity violation (APV) measurements. In Sec. $\nabla$ we describe the high energy data sets that we used in this analysis. In Sec. V1, we present our results on the fits and limits. In Sec. VII, we estimate the sensitivity in Run 2 of the Tevatron and at the LHC. A collection of data sets that we used in our analysis is placed in the appendix.

\section{INTERACTIONS OF THE KALUZA-KLEIN STATES}

In what follows, we use the formalism of Ref. [11], based on an extension of the SM to five dimensions, with the fifth dimension, $x^{5}$, compactified on the segment $S^{1} / Z_{2}$ (a circle of radius $R$ with the identification $\left.x^{5} \rightarrow-x^{5}\right)$. This segment has the length of $\pi R$. Two 3branes reside at the fixed points $x^{5}=0$ and $x^{5}=\pi R$. The SM gauge boson fields propagate in the 5D-bulk, while the SM fermions are confined to the 3-brane located at $x^{5}=0$. The Higgs sector consists of two Higgs doublets, $\phi_{1}$ and $\phi_{2}$ (with the ratio of vacuum expectation 
values $v_{2} / v_{1} \equiv \tan \beta$ ), which live in the bulk and on the SM brane, respectively.

The 5D Lagrangian is given by

$$
\mathcal{L}_{5}=-\frac{1}{4 g_{5}} F_{M N}^{2}+\left|D_{M} \phi_{1}\right|^{2}+\left(i \bar{\psi} \sigma^{\mu} D_{\mu} \psi+\left|D_{\mu} \phi_{2}\right|^{2}\right) \delta\left(x^{5}\right),
$$

where $D_{M}=\partial_{M}+i V_{M}, M=(\mu, 5)=(1, \ldots, 5)$, and $g_{5}$ is the $5 \mathrm{D}$ gauge coupling for the gauge boson $V$. Compactifying the fifth dimension on $S^{1} / Z_{2}$ with the expansion

$$
\Phi\left(x^{\mu}, x^{5}\right)=\sum_{n=0}^{\infty} \cos \left(\frac{n x^{5}}{R}\right) \Phi^{(n)}\left(x^{\mu}\right),
$$

the 4D Lagrangian becomes [11

$$
\begin{aligned}
\mathcal{L}_{4}= & \sum_{n=0}^{\infty}\left[-\frac{1}{4} F_{\mu \nu}^{(n) 2}+\frac{1}{2}\left(\frac{n^{2}}{R}+2 g^{2}\left|\phi_{1}\right|^{2}\right) V_{\mu}^{(n)} V^{(n) \mu}\right]+g^{2}\left|\phi_{2}\right|^{2}\left(V_{\mu}^{(0)}+\sqrt{2} \sum_{n=1}^{\infty} V_{\mu}^{(n)}\right)^{2} \\
& +i \bar{\psi} \sigma^{\mu}\left[\partial_{\mu}+i g V_{\mu}^{(0)}+i g \sqrt{2} \sum_{n=1}^{\infty} V_{\mu}^{(n)}\right] \psi+\ldots
\end{aligned}
$$

where $g=g_{5} / \sqrt{\pi R}$ is the $4 \mathrm{D}$ gauge coupling for the gauge boson $V$.

In the case of $\mathrm{SU}(2)_{L} \times \mathrm{U}(1)_{Y}$ symmetry, the charged-current $(\mathrm{CC})$ and neutral-current (NC) interactions after compactifying the fifth dimension are given by [14:

$$
\begin{aligned}
\mathcal{L}^{\mathrm{CC}} & =\frac{g^{2} v^{2}}{8}\left[W_{1}^{2}+\cos ^{2} \beta \sum_{n=1}^{\infty}\left(W_{1}^{(n)}\right)^{2}+2 \sqrt{2} \sin ^{2} \beta W_{1} \sum_{n=1}^{\infty} W_{1}^{(n)}+2 \sin ^{2} \beta\left(\sum_{n=1}^{\infty} W_{1}^{(n)}\right)^{2}\right] \\
& +\frac{1}{2} \sum_{n=1}^{\infty} n^{2} M_{C}^{2}\left(W_{1}^{(n)}\right)^{2}-g\left(W_{1}^{\mu}+\sqrt{2} \sum_{n=1}^{\infty} W_{1}^{(n) \mu}\right) J_{\mu}^{1}+(1 \rightarrow 2) \\
\mathcal{L}^{\mathrm{NC}} & =\frac{g v^{2}}{8 c_{\theta}^{2}}\left[Z^{2}+\cos ^{2} \beta \sum_{n=1}^{\infty}\left(Z^{(n)}\right)^{2}+2 \sqrt{2} \sin ^{2} \beta Z \sum_{n=1}^{\infty} Z^{(n)}+2 \sin ^{2} \beta\left(\sum_{n=1}^{\infty} Z^{(n)}\right)^{2}\right. \\
& +\frac{1}{2} \sum_{n=1}^{\infty} n^{2} M_{C}^{2}\left[\left(Z^{(n)}\right)^{2}+\left(A^{(n)}\right)^{2}\right] \\
& -\frac{e}{s_{\theta} c_{\theta}}\left(Z^{\mu}+\sqrt{2} \sum_{n=1}^{\infty} Z^{(n) \mu}\right) J_{\mu}^{Z}-e\left(A^{\mu}+\sqrt{2} \sum_{n=1}^{\infty} A^{(n) \mu}\right) J_{\mu}^{\mathrm{em}},
\end{aligned}
$$

where the fermion currents are:

$$
\begin{aligned}
J_{\mu}^{1,2} & =\bar{\psi}_{L} \gamma_{\mu}\left(\frac{\tau_{1,2}}{2}\right) \psi_{L}, \\
J_{\mu}^{Z} & =\bar{\psi} \gamma_{\mu}\left(g_{v}-\gamma^{5} g_{a}\right) \psi, \\
J_{\mu}^{\mathrm{em}} & =\bar{\psi} \gamma_{\mu} Q_{\psi} \psi,
\end{aligned}
$$

and $\left\langle\phi_{1}\right\rangle=v \cos \beta,\left\langle\phi_{2}\right\rangle=v \sin \beta ; g$ and $g^{\prime}$ are the gauge couplings of the $\mathrm{SU}(2)_{L}$ and $\mathrm{U}(1)_{Y}$, respectively; $g_{v}=T_{3 L} / 2-s_{\theta}^{2} Q$ and $g_{a}=T_{3 L} / 2$. Here, we used the following shorthand notations: $s_{\theta} \equiv \sin \theta_{W}$ and $c_{\theta} \equiv \cos \theta_{W}$, where $\theta_{W}$ is the weak-mixing angle. The 
tree-level (non-physical) $W$ and $Z$ masses are $M_{W}=g v / 2$ and $M_{Z}=M_{W} / c_{\theta}$. Since the compactification scale $M_{C}$ is expected to be in the $\mathrm{TeV}$ range, we therefore ignore in the above equations the mass of the zeroth-state gauge boson in the expression for the mass of the $n$-th KK excitation: $M_{n}=\sqrt{M_{0}^{2}+n^{2} M_{C}^{2}} \approx n M_{C}, n=1,2, \ldots$

Using the above Lagrangians we can describe the two major effects of the KK states: mixing with the SM gauge bosons and virtual exchanges in high-energy interactions.

\section{A. Mixing with the SM Gauge Bosons}

The first few terms in the Eqs. (2) and (3) imply the existence of mixings among the SM boson $(V)$ and its KK excitations $\left(V^{(1)}, V^{(2)}, \ldots\right)$ where $V=W, Z$. There is no mixing for the $A^{\mu}$ fields because of the $\mathrm{U}(1)_{\mathrm{EM}}$ symmetry. These mixings modify the electroweak observables (similar to the mixing between the $Z$ and $Z^{\prime}$ ). The SM weak eigenstate of the $Z$-boson, $Z^{(0)}$, mixes with its excited KK states $Z^{(n)}(n=1,2, \ldots)$ via a series of mixing angles, which depend on the masses of $Z^{(n)}, n=0,1, \ldots$ and on the angle $\beta$. The $Z$ boson studied at LEP 1 is then the lowest mass eigenstate after mixing. The couplings of the $Z^{(0)}$ to fermions are also modified through the mixing angles. The observables at LEP 1 can place strong constraints on the mixing, and thus on the compactification scale $M_{C}$. Similarly, the properties of the $W$ boson are also modified. However, so far the mass and couplings of the $W$ are not measured as precisely as the $Z$ observables, so the constraints on $M_{C}$ coming from the $W$ are weaker than those from the $Z$.

The effects on electroweak precision measurements have been previously studied [12, 13, 14, 15, 16, 17, 18]; we will summarize their results in the next section.

\section{B. Virtual Exchanges}

If the available energy is higher than the compactification scale the on-shell production of the Kaluza-Klein excitations of the gauge bosons can be observed [19]. However, for the present collider energies only indirect effects can be seen, as the compactification scale is believed to be at least a few $\mathrm{TeV}$. These indirect effects are due to virtual exchange of the KK-states.

When considering these virtual exchanges, we ignore a slight modification of the coupling 
constants to fermions due to the mixings among the KK states and so we use Eqs. (2) and (3) without the mixings ${ }^{1}$. This implies that any Feynman diagram which has an exchange of a $W, Z, \gamma$, or $g$ will be replicated for every corresponding KK state with the masses $n M_{C}$, where $n=1,2, \ldots$. Note that the coupling constant of the KK states to fermions is a factor of $\sqrt{2}$ larger than that for the corresponding SM gauge boson, due to the normalization of the KK excitations.

It has been shown in Ref. [10] that in the presence of the KK states of gauge bosons in the bulk, the renormalization-group evolution of the gauge couplings changes from the normal logarithmic running to a power running for energy scales above $M_{C}$. However, the energy scale of the processes that we consider in this paper is well below $M_{C}$. Consequently, the running of gauge couplings is the same as the normal logarithmic running in the SM [10]. Besides, we are not concerned about the additional real scalars transforming in the adjoint of each gauge group that are required to give masses to the gauge bosons [10]. This is because the scalars usually couple to light fermions via very small Yukawa couplings.

We start with Drell-Yan production of a pair of leptons. The amplitude squared for $q \bar{q} \rightarrow \ell^{+} \ell^{-}$or $\ell^{+} \ell^{-} \rightarrow q \bar{q}$ (without averaging over the initial spins or colors) is given by:

$$
\sum|\mathcal{M}|^{2}=4 u^{2}\left(\left|M_{\mathrm{LL}}^{\ell q}(s)\right|^{2}+\left|M_{\mathrm{RR}}^{\ell q}(s)\right|^{2}\right)+4 t^{2}\left(\left|M_{\mathrm{LR}}^{\ell q}(s)\right|^{2}+\left|M_{\mathrm{RL}}^{\ell q}(s)\right|^{2}\right),
$$

where

$$
\begin{aligned}
M_{\alpha \beta}^{\ell q}(s) & =e^{2}\left\{\frac{Q_{\ell} Q_{q}}{s}+\frac{g_{\alpha}^{\ell} g_{\beta}^{q}}{\sin ^{2} \theta_{W} \cos ^{2} \theta_{\mathrm{w}}} \frac{1}{s-M_{Z}^{2}}\right. \\
& \left.+2 \sum_{n=1}^{\infty}\left[\frac{Q_{\ell} Q_{q}}{s-n^{2} M_{C}^{2}}+\frac{g_{\alpha}^{\ell} g_{\beta}^{q}}{\sin ^{2} \theta_{W} \cos ^{2} \theta_{W}} \frac{1}{s-n^{2} M_{C}^{2}}\right]\right\} .
\end{aligned}
$$

Here $s, t, u$ are the usual Mandelstam variables, $g_{L}^{f}=T_{3 f}-Q_{f} \sin ^{2} \theta_{W}, g_{R}^{f}=-Q_{f} \sin ^{2} \theta_{\mathrm{w}}$, and $Q_{f}$ is the electric charge of the fermion $f$ in units of proton charge.

If the compactification scale $M_{C} \gg \sqrt{s}, \sqrt{|t|}, \sqrt{|u|}$, the above can further be simplified to:

$$
M_{\alpha \beta}^{\ell q}(s)=e^{2}\left\{\frac{Q_{\ell} Q_{q}}{s}+\frac{g_{\alpha}^{\ell} g_{\beta}^{q}}{\sin ^{2} \theta_{W} \cos ^{2} \theta_{W}} \frac{1}{s-M_{Z}^{2}}-\left(Q_{\ell} Q_{q}+\frac{g_{\alpha}^{\ell} g_{\beta}^{q}}{\sin ^{2} \theta_{W} \cos ^{2} \theta_{W}}\right) \frac{\pi^{2}}{3 M_{C}^{2}}\right\} .
$$

Based on the above formula the amplitude squared for deep-inelastic scattering at HERA can be obtained by a simple interchange of the Mandelstam variables. In the later section, we will derive the expressions for specific observables used in our analysis.

\footnotetext{
${ }^{1}$ Since $M_{C}>>M_{Z}$, the mixings are very small. Furthermore, they completely vanish for $\beta=0$.
} 


\section{REVIEW OF THE LOW-ENERGY CONSTRAINTS}

The effects of KK excitations in the low-energy limit can be included by eliminating their fields using equations of motion following from the Lagrangians given by Eqs. (2) and (3) [14:

$$
\begin{aligned}
W_{1,2}^{(n)} & =-\sqrt{2} \frac{\sin ^{2} \beta M_{W}^{2}}{n^{2} M_{C}^{2}} W_{1,2}+\sqrt{2} \frac{g}{n^{2} M_{C}^{2}} J^{1,2}+O\left(1 / M_{C}^{4}\right), \\
Z^{(n)} & =-\sqrt{2} \frac{\sin ^{2} \beta M_{Z}^{2}}{n^{2} M_{C}^{2}} Z+\sqrt{2} \frac{e}{s_{\theta} c_{\theta}} \frac{1}{n^{2} M_{C}^{2}} J^{Z}+O\left(1 / M_{C}^{4}\right), \\
A^{(n)} & =\sqrt{2} \frac{e}{n^{2} M_{C}^{2}} J^{\mathrm{em}}+O\left(1 / M_{C}^{4}\right) .
\end{aligned}
$$

Substituting back into Eqs. (2) and (3) we obtain the physical $W$ and $Z$ masses and the interaction Lagrangian [14]:

$$
\begin{aligned}
M_{W}^{2}= & M_{W}^{2}\left(1-c_{\theta}^{2} \sin _{\beta}^{4} X\right), \\
M_{Z}^{2}= & M_{Z}^{2}\left(1-\sin _{\beta}^{4} X\right), \\
\mathcal{L}_{\text {int }}^{\mathrm{CC}}= & -g J_{\mu}^{1} W^{1 \mu}\left(1-\sin ^{2} \beta c_{\theta}^{2} X\right)-\frac{g^{2}}{2 M_{Z}^{2}} X J_{\mu}^{1} J^{1 \mu}+(1 \rightarrow 2), \\
\mathcal{L}_{\text {int }}^{\mathrm{NC}}= & -\frac{e}{s_{\theta} c_{\theta}} J_{\mu}^{Z} Z^{\mu}\left(1-\sin ^{2} \beta X\right)-\frac{e^{2}}{2 s_{\theta}^{2} c_{\theta}^{2} M_{Z}^{2}} X J_{\mu}^{Z} J^{Z \mu} \\
& -e J_{\mu}^{\mathrm{em}} A^{\mu}-\frac{e^{2}}{2 M_{Z}^{2}} X J_{\mu}^{\mathrm{em}} J^{\mathrm{em} \mu}, \\
X= & \frac{\pi^{2} M_{Z}^{2}}{3 M_{C}^{2}} .
\end{aligned}
$$

The above low-energy Lagrangian already includes the effects of gauge-boson mixings and of virtual exchange of the KK states and thus can be used to calculate the precision observables. We illustrate this with a few examples. Using Eq. (5) we can calculate $G_{F}$ in muon decay:

$$
G_{F}=\frac{\sqrt{2} g^{2}}{8 M_{W}^{2}}\left(1+c_{\theta}^{2} X\right)\left(1-2 \sin ^{2} \beta c_{\theta}^{2} X\right) .
$$

Analogously, the partial width of the $Z$ boson into a pair of fermions can be calculated using Eq. (6):

$$
\Gamma(Z \rightarrow f \bar{f})=\frac{N_{c} M_{Z}}{12 \pi} \frac{e^{2}}{s_{\theta}^{2} c_{\theta}^{2}}\left(1-2 \sin ^{2} \beta X\right)\left(g_{v}^{2}+g_{a}^{2}\right),
$$

where $N_{c}=1$ (3) for leptons (quarks). Other quantities can be derived similarly.

In the following, we summarize the results presented in Refs. [12, 13, 14, 15, 16, 17, 18]. Nath and Yamaguchi [12] used data on $G_{F}, M_{W}$, and $M_{Z}$ and set the lower limit on $M_{C} \gtrsim$ 
1.6 TeV. Carone [16] studied a number of precision observables, such as $G_{F}, \rho, Q_{W}$, leptonic and hadronic widths of the $Z$. The most stringent constraint on $M_{C}$ comes from the hadronic width of the $Z: M_{C}>3.85 \mathrm{TeV}$. Strumia 15 obtained a limit $M_{C}>3.4-4.3 \mathrm{TeV}$ from a set of electroweak precision observables. Casalbuoni et al. [14] used the complete set of precision measurements, as well as $Q_{W}$ and $R_{\nu}$ 's from $\nu$-N scattering experiments, and obtained a limit $M_{C}>3.6 \mathrm{TeV}$. Rizzo and Wells [13] used the same set of data as the previous authors and obtained a limit $M_{C}>3.8 \mathrm{TeV}$. Cornet et al. [18 used the unitarity of the CKM matrix elements and were able to obtain a limit $M_{C}>3.3 \mathrm{TeV}$. Delgado et al. [17] studied a scenario in which quarks of different families are separated in the extra spatial dimension and set the limit $M_{C}>5 \mathrm{TeV}$ in this scenario.

\section{ATOMIC PARITY VIOLATION}

The 1999 atomic parity violation (APV) measurement on cesium 20] has drawn a great deal of attention because the data showed a $2.3 \sigma$ deviation from the SM prediction. Several explanations involving physics beyond the SM, such as extra $Z$ bosons [21] and leptoquarks [22], have been suggested. Later, however, the theoretical calculations used in the analysis had been questioned and new calculations appeared since [23]. As a result, data now agree with the SM prediction [24]:

$$
\Delta Q_{W} \equiv Q_{W}(\mathrm{Cs})-Q_{W}^{\mathrm{SM}}(\mathrm{Cs})=0.44 \pm 0.44 .
$$

The KK states of the $Z$ boson act similarly to a large number of extra $Z$ bosons with the same chiral couplings as the SM $Z$ boson. These KK states result in a non-zero $\Delta Q_{W}$.

The change in $Q_{W}$ due to the KK states of the $Z$ in terms of chiral couplings is given by 222:

$$
\begin{aligned}
\Delta Q_{W} & =\left(-11.4 \mathrm{TeV}^{2}\right)\left(\frac{-e^{2}}{\sin ^{2} \theta_{W} \cos ^{2} \theta_{W}}\right)\left(-g_{L}^{e}+g_{R}^{e}\right)\left(g_{L}^{u}+g_{R}^{u}\right) \eta \\
& +\left(-12.8 \mathrm{TeV}^{2}\right)\left(\frac{-e^{2}}{\sin ^{2} \theta_{W} \cos ^{2} \theta_{W}}\right)\left(-g_{L}^{e}+g_{R}^{e}\right)\left(g_{L}^{d}+g_{R}^{d}\right) \eta \\
& \approx\left(-0.6 \mathrm{TeV}^{2}\right) \eta
\end{aligned}
$$

where $\eta=\pi^{2} /\left(3 M_{C}^{2}\right), g_{L, R}^{f}=T_{3 f}-Q_{f} \sin ^{2} \theta_{W}$, and $\theta_{W}$ is the weak mixing angle. As seen from Eq. (7), the KK states with the same chiral couplings as the SM $Z$ boson give negative contributions to $Q_{W}$ 's, and therefore are disfavored by the data. 


\section{HIGH ENERGY PROCESSES AND DATA SETS}

Before describing the data sets used in our analysis, let us first specify certain important aspects of the analysis technique. Since the next-to-leading order (NLO) calculations do not exist for the new interactions yet, we use leading order (LO) calculations for contributions both from the SM and from new interactions, for consistency. However, in many cases, e.g. in the analysis of precision electroweak parameters, it is important to use the best available calculations of their SM values, as in many cases data is sensitive to the nextto-leading and sometimes even to higher-order corrections. Therefore, we normalize our leading order calculations to either the best calculations available, or to the low- $Q^{2}$ region of the data set, where the contribution from the KK states is expected to be vanishing. This is equivalent to introducing a $Q^{2}$-dependent $K$-factor and using the same $K$-factor for both the SM contribution and the effects of the KK resonances, which is well justified by the similarity between these extra resonances and the corresponding ground-state gauge boson. The details of this procedure for each data set are given in the corresponding section. Wherever parton distribution functions (PDFs) are needed, we use the CTEQ5L (leading order fit) set [25]. The reason to use the LO PDF set is that LO PDFs are extracted using LO cross section calculations, thus making them more consistent with our approach.

\section{A. HERA Neutral and Charged Current Data}

ZEUS [26] and H1 [27] have published results on the neutral-current (NC) and chargedcurrent (CC) deep-inelastic scattering (DIS) in $e^{+} p$ collisions at $\sqrt{s} \approx 300 \mathrm{GeV}$. The data sets collected by $\mathrm{H} 1$ and ZEUS correspond to an integrated luminosities of 35.6 and 47.7 $\mathrm{pb}^{-1}$, respectively. $\mathrm{H} 1$ [27] has also published $\mathrm{NC}$ and $\mathrm{CC}$ analysis for the most recent data collected in $e^{-} p$ collisions at $\sqrt{s} \approx 320 \mathrm{GeV}$ with an integrated luminosity of $16.4 \mathrm{pb}^{-1}$.

We used single-differential cross sections $d \sigma / d Q^{2}$ presented by ZEUS [26] and doubledifferential cross sections $d^{2} \sigma / d x d Q^{2}$ published by $\mathrm{H} 1$ [27]. The double-differential cross section for NC DIS in the $e^{+} p$ collisions, including the effect of the KK states of the $\gamma$ and $Z$, is given by

$$
\frac{d^{2} \sigma}{d x d Q^{2}}\left(e^{+} p \rightarrow e^{+} X\right)=
$$




$$
\begin{aligned}
& \frac{1}{16 \pi}\left\{\sum_{q} f_{q}(x)\left[(1-y)^{2}\left(\left|M_{L L}^{e q}(t)\right|^{2}+\left|M_{R R}^{e q}(t)\right|^{2}\right)+\left|M_{L R}^{e q}(t)\right|^{2}+\left|M_{R L}^{e q}(t)\right|^{2}\right]\right. \\
& \left.+\sum_{\bar{q}} f_{\bar{q}}(x)\left[\left|M_{L L}^{e q}(t)\right|^{2}+\left|M_{R R}^{e q}(t)\right|^{2}+(1-y)^{2}\left(\left|M_{L R}^{e q}(t)\right|^{2}+\left|M_{R L}^{e q}(t)\right|^{2}\right)\right]\right\}
\end{aligned}
$$

where $Q^{2}=s x y$ is the square of the momentum transfer and $f_{q / \bar{q}}(x)$ are parton distribution functions. The reduced amplitudes $M_{\alpha \beta}^{e q}$ are given by Eq. (4). The double differential cross section for CC DIS, including the effect of KK states of $W$, can be written as

$$
\frac{d^{2} \sigma}{d x d Q^{2}}\left(e^{+} p \rightarrow \bar{\nu} X\right)=\frac{g^{4}}{64 \pi}\left|\frac{1}{-Q^{2}-M_{W}^{2}}-\frac{\pi^{2}}{3 M_{C}^{2}}\right|^{2}\left[(1-y)^{2}(d(x)+s(x))+\bar{u}(x)+\bar{c}(x)\right],
$$

where $d(x), s(x), \bar{u}(x), \bar{c}(x)$ are the parton distribution functions. The single differential cross section $d \sigma / d Q^{2}$ is obtained from the above equations by integrating over $x$. The cross section in the $e^{-} p$ collisions can be obtained by interchanging $(L L \leftrightarrow L R, R R \leftrightarrow R L)$ in Eq. (8) and by interchanging $(q(x) \leftrightarrow \bar{q}(x))$ in Eq. (9).

We normalize the tree-level SM cross section to that measured in the low- $Q^{2}\left(Q^{2} \lesssim 2000\right.$ $\left.\mathrm{GeV}^{2}\right)$ data by a scale factor $C$ ( $C$ is very close to 1 numerically). The cross section $\sigma$ used in the fitting procedure is given by

$$
\sigma=C\left(\sigma_{\mathrm{SM}}+\sigma_{\text {interf }}+\sigma_{\mathrm{KK}}\right)
$$

where $\sigma_{\text {interf }}$ is the interference term between the SM and the KK states and $\sigma_{\mathrm{KK}}$ is the cross section due to the KK-state interactions only.

\section{B. Drell-Yan Production at the Tevatron}

Both CDF [28] and DØ [29] measured the differential cross section $d \sigma / d M_{\ell \ell}$ for Drell-Yan production, where $M_{\ell \ell}$ is the invariant mass of the lepton pair. (CDF analyzed data in both the electron and muon channels; DØ analyzed only the electron channel.)

The differential cross section, including the contributions from the KK states of the photon and $Z$, is given by

$$
\frac{d^{2} \sigma}{d M_{\ell \ell} d y}=K \frac{M_{\ell \ell}^{3}}{72 \pi s} \sum_{q} f_{q}\left(x_{1}\right) f_{\bar{q}}\left(x_{2}\right)\left(\left|M_{L L}^{e q}(\hat{s})\right|^{2}+\left|M_{L R}^{e q}(\hat{s})\right|^{2}+\left|M_{R L}^{e q}(\hat{s})\right|^{2}+\left|M_{R R}^{e q}(\hat{s})\right|^{2}\right)
$$

where $M_{\alpha \beta}^{e q}$ is given by Eq. (丑), $\hat{s}=M_{\ell \ell}^{2}, \sqrt{s}$ is the center-of-mass energy in the $p \bar{p}$ collisions, $M_{\ell \ell}$ and $y$ are the invariant mass and the rapidity of the lepton pair, respectively, and $x_{1,2}=$ 
$\frac{M_{\ell \ell}}{\sqrt{s}} e^{ \pm y}$. The variable $y$ is integrated numerically to obtain the invariant mass spectrum. The QCD $K$-factor is given by $K=1+\frac{\alpha_{s}(\hat{s})}{2 \pi} \frac{4}{3}\left(1+\frac{4 \pi^{2}}{3}\right)$. We scale this tree-level SM cross section by normalizing it to the $Z$-peak cross section measured with the data. The cross section used in the fitting procedure is then obtained similarly to that in Eq. (10).

\section{LEP 2 Data}

We analyze LEP 2 observables sensitive to the effects of the KK states of the photon and $Z$, including hadronic and leptonic cross sections and forward-backward asymmetries. The LEP Electroweak Working Group combined the $q \bar{q}, \mu^{+} \mu^{-}$, and $\tau^{+} \tau^{-}$data from all four LEP collaborations [30 for the machine energies between 130 and $202 \mathrm{GeV}$. We use the following quantities in our analysis: (i) total hadronic cross sections; (ii) total $\mu^{+} \mu^{-}, \tau^{+} \tau^{-}$ cross sections; (iii) forward-backward asymmetries in the $\mu$ and $\tau$ channels; and (iv) ratio of $b$-quark and $c$-quark production to the total hadronic cross section, $R_{b}$ and $R_{c}$. We take into account the correlations of the data points in each data set as given by [30.

For other channels we use various data sets from individual experiments. They are [31, 32, 33, 34: (i) Bhabha scattering cross section $\sigma\left(e^{+} e^{-} \rightarrow e^{+} e^{-}\right)$; (ii) angular distribution or forward-backward asymmetry in hadroproduction $e^{+} e^{-} \rightarrow q \bar{q}$; (iii) angular distribution or forward-backward asymmetry in the $e^{+} e^{-}, \mu^{+} \mu^{-}$, and $\tau^{+} \tau^{-}$production.

The angular distribution for $e^{-} e^{+} \rightarrow f \bar{f}(f=q, e, \mu, \tau)$ is given by

$$
\begin{aligned}
& \frac{d \sigma}{d \cos \theta}= \\
& \frac{N_{f} s}{128 \pi}\left\{(1+\cos \theta)^{2}\left(\left|M_{L L}^{e f}(s)\right|^{2}+\left|M_{R R}^{e f}(s)\right|^{2}\right)+(1-\cos \theta)^{2}\left(\left|M_{L R}^{e f}(s)\right|^{2}+\left|M_{R L}^{e f}(s)\right|^{2}\right)\right. \\
& +\delta_{e f}\left[(1+\cos \theta)^{2}\left(\left|M_{L L}^{e f}(s)+M_{L L}^{e f}(t)\right|^{2}+\left|M_{R R}^{e f}(s)+M_{R R}^{e f}(t)\right|^{2}-\left|M_{L L}^{e f}(s)\right|^{2}-\left|M_{R R}^{e f}(s)\right|^{2}\right)\right. \\
& \left.\left.+4\left(\left|M_{L R}^{e f}(t)\right|^{2}+\left|M_{R L}^{e f}(t)\right|^{2}\right)\right]\right\}
\end{aligned}
$$

where $N_{f}=1(3)$ for $\ell(q)$, and $M_{\alpha \beta}^{e f}$ is given by Eq. (四). The additional terms for $f=e$ arise from the $t, u$-channel exchange diagrams.

To minimize the uncertainties from higher-order corrections, we normalize the tree-level SM calculations to the NLO cross section, quoted in the corresponding experimental papers. We then scale our tree-level results, including contributions from the KK states of the $Z$ and 
$\gamma$, with this normalization factor, similar to Eq. (10). When fitting angular distribution, we fit to the shape only, and treat the normalization as a free parameter of the fit.

\section{Kaluza-Klein states of the Gluon in the Dijet Production at the Tevatron}

Since the gauge bosons propagate in extra dimensions, the Kaluza-Klein momentum conservation applies at their self-coupling vertices. Because of this conservation, the triple interaction vertex with two gluons on the SM 3-brane and one KK state of the gluon in the bulk vanishes. (However, the quartic vertex with two gluons on the SM 3-brane and two gluon KK states in the bulk does exist.) That is why the Lagrangian in Eq. (1) only has the interactions of KK states of the gluon with fermions, but not with gluons. (Furthermore, if we treated the trilinear interaction between the gluons and the KK states of the gluon the same as the SM triple-gluon interaction, the gauge invariance would be violated at the order of $\left(1 / M_{C}^{2}\right)$.)

The formulas for dijet production, including the contributions from KK states of the gluon (summed over the final-state and averaged over the initial-state helicities and colors), are:

$$
\begin{aligned}
\bar{\sum}\left|\mathcal{M}\left(q q^{\prime} \rightarrow q q^{\prime}\right)\right|^{2}= & \frac{4}{9} g_{s}^{4}\left(\hat{s}^{2}+\hat{u}^{2}\right)\left(\frac{1}{\hat{t}}-\frac{\pi^{2}}{3 M_{C}^{2}}\right)^{2}, \\
\bar{\sum}|\mathcal{M}(q q \rightarrow q q)|^{2}= & g_{s}^{4}\left[\frac{4}{9}\left(\hat{s}^{2}+\hat{u}^{2}\right)\left(\frac{1}{\hat{t}}-\frac{\pi^{2}}{3 M_{C}^{2}}\right)^{2}+\frac{4}{9}\left(\hat{s}^{2}+\hat{t}^{2}\right)\left(\frac{1}{\hat{u}}-\frac{\pi^{2}}{3 M_{C}^{2}}\right)^{2}\right. \\
& \left.-\frac{8}{27} \hat{s}^{2}\left(\frac{1}{t}-\frac{\pi^{2}}{3 M_{C}^{2}}\right)\left(\frac{1}{\hat{u}}-\frac{\pi^{2}}{3 M_{C}^{2}}\right)\right], \\
\bar{\sum}\left|\mathcal{M}\left(q \bar{q} \rightarrow q^{\prime} \bar{q}^{\prime}\right)\right|^{2}= & \frac{4}{9} g_{s}^{4}\left(\hat{t}^{2}+\hat{u}^{2}\right)\left(\frac{1}{\hat{s}}-\frac{\pi^{2}}{3 M_{C}^{2}}\right)^{2}, \\
\bar{\sum}|\mathcal{M}(q \bar{q} \rightarrow q \bar{q})|^{2}= & g_{s}^{4}\left[\frac{4}{9}\left(\hat{t}^{2}+\hat{u}^{2}\right)\left(\frac{1}{\hat{s}}-\frac{\pi^{2}}{3 M_{C}^{2}}\right)^{2}+\frac{4}{9}\left(\hat{s}^{2}+\hat{u}^{2}\right)\left(\frac{1}{\hat{t}}-\frac{\pi^{2}}{3 M_{C}^{2}}\right)^{2}\right. \\
& \left.-\frac{8}{27} \hat{u}^{2}\left(\frac{1}{t}-\frac{\pi^{2}}{3 M_{C}^{2}}\right)\left(\frac{1}{\hat{s}}-\frac{\pi^{2}}{3 M_{C}^{2}}\right)\right], \\
\bar{\sum}|\mathcal{M}(q \bar{q} \rightarrow g g)|^{2}= & g_{s}^{4}\left\{\frac{32}{27} \frac{\hat{u}^{2}+\hat{t}^{2}}{\hat{u} \hat{t}}-\frac{8}{3} \frac{\hat{u}^{2}+\hat{t}^{2}}{\hat{s}^{2}}\right\}, \\
\bar{\sum}|\mathcal{M}(g g \rightarrow q \bar{q})|^{2}= & g_{s}^{4}\left\{\frac{1}{6} \frac{\hat{u}^{2}+\hat{t}^{2}}{\hat{u} \hat{t}}-\frac{3}{8} \frac{\hat{u}^{2}+\hat{t}^{2}}{\hat{s}^{2}}\right\}, \\
\bar{\sum}|\mathcal{M}(q g \rightarrow q g)|^{2}= & g_{s}^{4}\left\{\frac{\hat{s}^{2}+\hat{u}^{2}}{\hat{t}^{2}}-\frac{4}{9} \frac{\hat{s}^{2}+\hat{u}^{2}}{\hat{u}_{\hat{s}}}\right\},
\end{aligned}
$$




$$
\bar{\sum}|\mathcal{M}(g g \rightarrow g g)|^{2}=\frac{9}{4} g_{s}^{4}\left\{\frac{\hat{s}^{2}+\hat{u}^{2}}{\hat{t}^{2}}+\frac{\hat{s}^{2}+\hat{t}^{2}}{\hat{u}^{2}}+\frac{\hat{t}^{2}+\hat{u}^{2}}{\hat{s}^{2}}+3\right\} .
$$

In the above, if the final state particles are different, the corresponding equations need to be symmetrized via $u \leftrightarrow t$ substitution. The parton-level differential cross section is given by

$$
\frac{d \hat{\sigma}}{d \cos \theta^{*}}=\frac{1}{32 \pi \hat{s}} \bar{\sum}|\mathcal{M}|^{2},
$$

where the range of $\cos \theta^{*}$ is from 0 to 1 . This parton-level cross section is then convoluted with the parton distribution functions to give the total cross section. The above equations are reduced to the SM cross sections in the $M_{C} \rightarrow \infty$ limit. The last four equations are the same as the SM cross sections, because of the vanishing trilinear gluon vertex involving two ground-state gluons.

Both CDF 35] and DØ [36] published data on dijet production, including invariant mass $M_{j j}$ and angular distributions. In the fit, we take into account the full correlation of data points in the data sets, as given by each experiment. We normalize the tree-level SM dijet cross section to the low dijet invariant mass data, $M_{j j}<400 \mathrm{GeV}$.

Collider implications of the KK states of the gluon have also been considered recently in Ref. [37.

\section{E. Kaluza-Klein States of the Gluon in the $t \bar{t}$ Production at the Tevatron}

In Ref. [38], it was shown that the $t \bar{t}$ production in Run 2 of the Tevatron can be used to probe the compactification scales up to $\sim 3 \mathrm{TeV}$. In this paper, we consider the sensitivity from the existing Run 1 data by using the tree-level $t \bar{t}$ production cross section, including the contribution of the KK states of the gluon in the $q \bar{q} \rightarrow t \bar{t}$ channel. (The $g g \rightarrow t \bar{t}$ channel does not have the triple vertex interaction with two gluons from the SM 3-brane and one KK state of the gluon in the bulk, as explained in the previous subsection.)

The subprocess cross sections are given by

$$
\begin{aligned}
\frac{d \hat{\sigma}}{d \cos \theta^{*}}(q \bar{q} \rightarrow t \bar{t}) & =\frac{g_{s}^{4} \beta}{72 \pi \hat{s}}\left(\frac{1}{\hat{s}}-\frac{\pi^{2}}{3 M_{C}^{2}}\right)^{2}\left[\left(m_{t}^{2}-\hat{t}\right)^{2}+\left(m_{t}^{2}-\hat{u}\right)^{2}+2 \hat{s} m_{t}^{2}\right], \\
\frac{d \hat{\sigma}}{d \cos \theta^{*}}(g g \rightarrow t \bar{t}) & =\frac{g_{s}^{4} \beta}{768 \pi \hat{s}}\left\{\frac{4}{\left(\hat{t}-m_{t}^{2}\right)^{2}}\left(-m_{t}^{4}-3 m_{t}^{2} \hat{t}-m_{t}^{2} \hat{u}+\hat{u} \hat{t}\right)\right. \\
& +\frac{4}{\left(\hat{u}-m_{t}^{2}\right)^{2}}\left(-m_{t}^{4}-3 m_{t}^{2} \hat{u}-m_{t}^{2} \hat{t}+\hat{u} \hat{t}\right)
\end{aligned}
$$




$$
\begin{aligned}
& +\frac{m_{t}^{2}}{\left(\hat{u}-m_{t}^{2}\right)\left(\hat{t}-m_{t}^{2}\right)}\left(2 m_{t}^{2}+\hat{t}+\hat{u}\right)+18 \frac{1}{\hat{s}^{2}}\left(m_{t}^{4}-m_{t}^{2}(\hat{t}+\hat{u})+\hat{t} \hat{u}\right) \\
& \left.+\frac{9}{\hat{t}-m_{t}^{2}} \frac{1}{\hat{s}}\left(m_{t}^{4}-2 m_{t}^{2} \hat{t}+\hat{u} \hat{t}\right)+\frac{9}{\hat{u}-m_{t}^{2}} \frac{1}{\hat{s}}\left(m_{t}^{4}-2 m_{t}^{2} \hat{u}+\hat{u} \hat{t}\right)\right\}
\end{aligned}
$$

where $\beta=\sqrt{1-4 m_{t}^{2} / \hat{s}}$ and $\hat{s}, \hat{t}, \hat{u}$ are Mandelstam variables. The above cross section is reduced to the SM top pair production cross section in the $M_{C} \rightarrow \infty$ limit.

The latest theoretical calculations of the $t \bar{t}$ cross section, including higher-order contributions, at $\sqrt{s}=1.8 \mathrm{TeV}$ correspond to $4.7-5.5 \mathrm{pb}$ [39]. The present data on the $t \bar{t}$ cross sections are 40

$$
\begin{aligned}
\sigma_{t \bar{t}}(\mathrm{CDF}) & =6.5_{-1.4}^{+1.7 \mathrm{pb}} \\
\sigma_{t \bar{t}}(\mathrm{D} \varnothing) & =5.9 \pm 1.7 \mathrm{pb}
\end{aligned}
$$

and the top-quark mass measurements are

$$
\begin{aligned}
m_{t}(\mathrm{CDF}) & =176.1 \pm 6.6 \mathrm{GeV} \\
m_{t}(\mathrm{D} \varnothing) & =172.1 \pm 7.1 \mathrm{GeV}
\end{aligned}
$$

In our analysis, we normalize the tree-level SM cross section to the mean of the latest theoretical predictions $(5.1 \mathrm{pb})$, and use this normalization coefficient to predict the cross section in presence of the KK states of the gluon (similar to Eq. (10)).

The effects of KK states of the $W$ boson on single top production were recently considered in Ref. 41].

\section{CONSTRAINTS FROM HIGH ENERGY EXPERIMENTS}

In the previous section, we have described the data sets from various high energy experiments used in our analysis. Based on the above individual and combined data sets, we perform a fit to the sum of the SM prediction and the contribution of the KK states of gauge bosons, normalizing our tree-level cross section to the best available higher-order calculations, as explained above. As seen from Eq. (4), the effects of the KK states always enter the equations in the form $\pi^{2} /\left(3 M_{C}^{2}\right)$. Therefore, we parameterize these effects with a single fit parameter $\eta$ :

$$
\eta=\frac{\pi^{2}}{3 M_{C}^{2}} .
$$


In most cases, the differential cross sections in presence of the KK states of gauge bosons are bilinear in $\eta$.

The best-fit values of $\eta$ for each individual data set and their combinations are shown in Table [. In all cases, the preferred values from the fit are consistent with zero, and therefore we proceed with setting limits on $\eta$. The one-sided 95\% C.L. upper limit on $\eta$ is defined as:

$$
0.95=\frac{\int_{0}^{\eta_{95}} d \eta P(\eta)}{\int_{0}^{\infty} d \eta P(\eta)}
$$

where $P(\eta)$ is the fit likelihood function given by $P(\eta)=\exp \left(-\left(\chi^{2}(\eta)-\chi_{\min }^{2}\right) / 2\right)$. The corresponding upper 95\% C.L. limits on $\eta$ and lower $95 \%$ C.L. limits on $M_{C}$ are also shown in Table 1 .

\section{SENSITIVITY IN RUN 2 OF THE TEVATRON AND AT THE LHC}

At the Tevatron, the best channel to probe the KK states of photon or $Z$ boson is DrellYan production. Since the typical $\sqrt{\hat{s}}$ in Run 2 is well below the limit obtained in the

previous section, the approximation $M_{C}^{2} \gg \hat{s},|\hat{t}|,|\hat{u}|$ is still valid. Therefore, we can use the reduced amplitudes of Eq. (田). This approximation also holds well for the LHC, which was tested by a direct comparison of the approximate cross section given by Eq. (4) and exact sum over the KK resonances, for values of $M_{C} \sim 10 \mathrm{TeV}$.

In Ref. [42], we showed that using the double differential distribution $d^{2} \sigma / M_{\ell \ell} d \cos \theta$ can increase the sensitivity to the KK states of the graviton compared to the use of singledifferential distributions. Similarly, we expect this to be the case for the KK states of the photon and the $Z$ boson. The double differential cross section for Drell-Yan production, including the interactions of the KK states of the $\gamma$ and $Z$, is given by

$$
\begin{aligned}
\frac{d^{3} \sigma}{d M_{\ell \ell} d y d \cos \theta^{*}}= & K \sum_{q} \frac{M_{\ell \ell}^{3}}{192 \pi s} f_{q}\left(x_{1}\right) f_{\bar{q}}\left(x_{2}\right)\left[\left(1+\cos \theta^{*}\right)^{2}\left(\left|M_{L L}^{e q}(\hat{s})\right|^{2}+\left|M_{R R}^{e q}(\hat{s})\right|^{2}\right)\right. \\
& \left.+\left(1-\cos \theta^{*}\right)^{2}\left(\left|M_{L R}^{e q}(\hat{s})\right|^{2}+\left|M_{R L}^{e q}(\hat{s})\right|^{2}\right)\right]
\end{aligned}
$$

where $M_{\alpha \beta}^{e q}$ 's are given by Eq. (雨), $\theta^{*}$ is the scattering angle in the rest frame of the initial partons, $\hat{s}=M_{\ell \ell}^{2}, d x_{1} d x_{2}=\left(2 M_{\ell \ell} / s\right) d M_{\ell \ell} d y$, and $x_{1,2}=M_{\ell \ell} e^{ \pm y} / \sqrt{s}$.

We follow the prescription of Ref. [42 and use the Bayesian approach, which correctly takes into account both the statistical and systematic uncertainties, in the estimation of the 
sensitivity to $\eta \equiv \pi^{2} /\left(3 M_{C}^{2}\right) .{ }^{2}$ Due to the high statistics in Run 2 and particularly at the LHC, the overall systematics becomes dominated by the systematics on the $\hat{s}$-dependence of the $K$-factor from the NLO corrections. (Systematic uncertainties on the integrated luminosity and efficiencies are not as important as before, because they get canceled out when normalizing the tree level SM cross section to the $Z$-peak region in the data.) The uncertainty on the $K$-factor from the NLO calculations for Drell-Yan production [43] is currently known to a $3 \%$ level, so we use this as the correlated systematics in our calculations on $M_{C}$. For the LHC we quote the limits for the same nominal $3 \%$ uncertainty and also show how the sensitivity improves if the uncertainty on the $K$-factor shape is reduced to a $1 \%$ level. It shows the importance of higher-order calculations of the Drell-Yan cross section, which we hope will become available by the time the LHC turns on. ${ }^{3}$

In the simulation, we use a dilepton efficiency of $90 \%$, a rapidity coverage of $|\eta|<2.0$, and typical energy resolutions of the Tevatron or LHC experiments. The simulation is done for a single collider experiment in the combination of the dielectron and dimuon channels.

As expected, the fit to double-differential cross sections yields a $\sim 10 \%$ better sensitivity to $M_{C}$ than just using one-dimensional differential cross sections. We illustrate this by calculating the sensitivity to $M_{C}$ in Run 1, which is slightly higher than the result obtained from the fit to the invariant mass spectrum from CDF and D $\varnothing$.

The sensitivity, at the $95 \%$ C.L., to $M_{C}$ in Run $1\left(120 \mathrm{pb}^{-1}\right)$, Run $2 \mathrm{a}\left(2 \mathrm{fb}^{-1}\right)$, Run $2 \mathrm{~b}$ $\left(15 \mathrm{fb}^{-1}\right)$, and at the LHC $\left(100 \mathrm{fb}^{-1}\right)$ is given in Table $\mathbb{1 1}$. While the Run 2 sensitivity is somewhat inferior to the current indirect limits from precision electroweak data, LHC would offer a significantly higher sensitivity to $M_{C}$, well above $10 \mathrm{TeV}$.

When this work is completed, we learned of a preliminary study on a similar topic for the LHC [45], which yielded a somewhat lower sensitivity. Very recently, a complementary paper [46] on the effects of KK excitations of gauge bosons at high-energy $e^{+} e^{-}$colliders has appeared in LANL archives.

\footnotetext{
${ }^{2}$ Note that the maximum likelihood method, as given by Eq. (11), artificially yields $10 \%$ higher sensitivity to $M_{C}$, as it does not properly treat the cases when the likelihood maximum is found in the unphysical region $\eta<0$.

3 The electroweak radiative corrections have recently been computed in Ref. 44].
} 


\section{Acknowledgments}

We would like to thank Ignatios Antoniadis, Keith Dienes, JoAnne Hewett, Steve Mrenna, Giacomo Polesello, and Tom Rizzo for useful discussions. This research was partially supported by the U.S. Department of Energy under Grants No. DE-FG02-91ER40688 and by A.P. Sloan Foundation, and by the National Center for Theoretical Science under a grant from the National Science Council of Taiwan R.O.C.

\section{Appendix}

Tables [II to XXII are the data sets that we used in our analysis.

[1] T. Kaluza, Preuss. Akad. Wiss. (1921) 966; O. Klein, Z. Phys. 37, 895 (1926).

[2] I. Antoniadis, Phys. Lett. B246, 377 (1990); J. Lykken, Phys. Rev. D 54, 3693 (1996); G. Shiu and S. Tye, Phys. Rev. D 58, 106007 (1998); I. Antoniadis and C. Bachas, Phys. Lett. B450, 83 (1999).

[3] N. Arkani-Hamed, S. Dimopoulos, G. Dvali, Phys. Lett. B429, 263 (1998); Phys. Rev. D 59, 086004 (1999).

[4] G. Giudice, R. Rattazzi, and J. Wells, Nucl. Phys. B544, 3 (1999) and revised version 2, e-print hep-ph/9811291.

[5] E.A. Mirabelli, M. Perelstein, and M.E. Peskin, Phys. Rev. Lett. 82, 2236 (1999).

[6] T. Han, J.D. Lykken, and R.-J. Zhang, Phys. Rev. D 59, 105006 (1999) and revised version 4, e-print hep-ph/9811350.

[7] J.L. Hewett, Phys. Rev. Lett. 82, 4765 (1999); K. Cheung, Phys. Lett. B460, 383 (1999).

[8] S. Dimopoulos and G. Landsberg, Phys. Rev. Lett. 87, 161602 (2001); S. Giddings and S. Thomas, e-print hep-ph/0106219v3; S. Dimopoulos and R. Emparan, e-print hepph/0108060; S. Hossenfelder et al., e-print hep-ph/0109085; K. Cheung, e-print hep$\mathrm{ph} / 0110163$.

[9] See, e.g, K. Cheung, in Proc. 7th International Symposium on Particles, Strings and Cosmology (PASCOS 99), Granlibakken, Tahoe City, California, 10-16 Dec 1999, e-print hep- 
ph/0003306; G. Landsberg, In Proc. XXXVIth Rencontres de Moriond "QCD and High Energy Hadronic Interactions" Conference, Les Arcs, March 17-24, 2001, e-print hep-ph/0105039.

[10] K. Dienes, E. Dudas, and T. Gherghetta, Nucl. Phys. B537, 47 (1999).

[11] A. Pomarol and M. Quirós, Phys. Lett. B438, 255 (1998); M. Masip and A. Pomarol, Phys. Rev. D 60, 096005 (1999); I. Antoniadis, K. Benakli, and M. Quirós, Phys. Lett. B460, 176 (1999).

[12] P. Nath and M. Yamaguchi, Phys. Rev. D 60, 116004 (1999); P. Nath, Y. Yamada, and M. Yamaguchi, Phys. Lett. B466, 100 (1999).

[13] T. Rizzo and J. Wells, Phys. Rev. D 61, 016007 (2000).

[14] R. Casalbuoni, S. Curtis, D. Dominici, and R. Gatto, Phys. Lett. B462, 48 (1999).

[15] A. Strumia, Phys. Lett. B466, 107 (1999).

[16] C. Carone, Phys. Rev. D 61, 015008 (2000).

[17] A. Delgado, A. Pomarol, and M. Quirós, JHEP 0001, 030 (2000).

[18] F. Cornet, M. Relaño, and J. Rico, Phys. Rev. D 61, 037701 (2000).

[19] T.G. Rizzo, Phys. Rev. D 61, 055005 (2000); H. Davoudiasl, J.L. Hewett, T.G. Rizzo, Phys. Rev. D 63, 075004 (2001).

[20] S.C. Bennett and C.E. Wieman, Phys. Rev. Lett. 82, 2484 (1999).

[21] G. Cho, Mod. Phys. Lett. A 15, 311 (2000); J. Rosner, Phys. Rev. D 61, 016006 (1999); R. Casalbuoni, S. De Curtis, D. Dominici, and R. Gatto, Phys. Lett. B460, 135 (1999); J. Erler and P. Langacker, Phys. Rev. Lett. 84, 212 (2000).

[22] V. Barger and K. Cheung, Phys. Lett. B480, 149 (2000).

[23] A. Derevianko, Phys. Rev. Lett. 85, 1618 (2000).

[24] P. Langacker, e-print hep-ph/0102085.

[25] H.L. Lai et al. (CTEQ Collaboration), Eur. Phys. J. C12, 375 (2000).

[26] J. Breitweg et al. (ZEUS Collaboration), Eur. Phys. J. C11, 427 (1999); Eur. Phys. J. C12, 411 (2000).

[27] C. Adloff et al. (H1 Collaboration), Eur. Phys. J. C13, 609 (2000); Eur. Phys. J. C19, 269 (2001).

[28] F. Abe et al. (CDF Collaboration), Phys. Rev. Lett. 79, 2198 (1997); also updates on the electron data sample at the following URL: http://www-cdf.fnal.gov/physics/ewk/ DY_dSdM_Afb/dSdM_Afb.html. 
[29] B. Abbott et al. (DØ Collaboration), Phys. Rev. Lett. 82, 4769 (1999).

[30] LEP Electroweak Working Group, “A Combination of Preliminary Electroweak Measurements and Constraints on the Standard Model", e-print hep-ex/0103048.

[31] R. Barate et al. (ALEPH Collaboration), Eur. Phys. J. C12, 183 (2000); ALEPH-99018, URL: http://alephwww.cern.ch/ALPUB/oldconf/oldconf99/20/qcd_winter.ps (unpublished); ALEPH-2000-047, URL: http://alephwww.cern.ch/ALPUB/oldconf/osaka00/ fermion/osaka_final.ps (unpublished); ALEPH-2001-019, URL: http://alephwww.cern. ch/ALPUB/oldconf/oldconf01/24/fermion.ps (unpublished).

[32] P. Abreu et al. (DELPHI Collaboration), Eur. Phys. J. C11, 383 (1999); Phys. Lett. B485, 45 (2000); A. Behrmann et al. (DELPHI Collaboration), DELPHI99-135, URL: http://delphiwww.cern.ch/ ${ }^{\sim} \mathrm{pubxx} /$ www/delsec/conferences/tampere99/ paper_6_362.ps.gz (unpublished); DELPHI-2000-128, URL: http://delphiwww.cern.ch/ 〜pubxx/www/delsec/delnote/public/2000_128_conf_427.ps.gz (unpublished).

[33] M. Acciarri, (L3 Collaboration), Phys. Lett. B370, 195 (1996); Phys. Lett. B407, 361 (1997); L3 Note 2398, URL: http://13www.cern.ch/conferences/EPS99/papers/note_2398.ps (unpublished); Phys. Lett. B479, 101 (2000).

[34] K. Ackerstaff et al. (OPAL Collaboration), Eur. Phys. J. C2, 441 (1998); Eur. Phys. J. C6, 1 (1999); G. Abbiendi et al. (OPAL Collaboration), Eur. Phys. J. C13, 553 (2000); OPAL Note PN424, URL: http://opal.web.cern.ch/Opal/pubs/physnote/html/pn424.html (unpublished).

[35] F. Abe et al. (CDF Collaboration), Phys. Rev. Lett. 77, 5336 (1996), Erratum ibid. 78, 4307 (1997); Phys. Rev. D 61, 091101 (2000).

[36] B. Abbott et al. (DØ Collaboration), Phys. Rev. Lett. 80, 666 (1998); Phys. Rev. Lett. 82, 2457 (1999).

[37] D. Dicus, C. McMullen, S. Nandi, e-print hep-ph/0012259.

[38] J. Lykken and S. Nandi, Phys. Lett. B485, 224 (2000).

[39] E. Berger and H. Contopanagis, Phys. Rev. D 54, 3085 (1996); S. Catani et al., Phys. Lett. B378, 329 (1996).

[40] S. Blusk (CDF Collaboration), in Proc. 30th International Conf. on High Energy Physics, ICHEP2000, Osaka, Japan, 27 July - 2 August 2000, edited by C.S. Lim, T. Yamanaka, p. 811; D. Chakraborty (DØ Collaboration), ibid., p. 814. 
[41] A. Datta, P.J. O’Donnell, Z.H. Lin, X. Zhang, and T. Huang, Phys. Lett. B483, 203 (2000).

[42] K. Cheung and G. Landsberg, Phys. Rev. D 62, 076003 (2000).

[43] R. Hamberg, W.L. Van Neerven, and T. Matsuura, Nucl. Phys. B359, 343 (1991).

[44] U. Baur, O. Brein, W. Hollik, C. Schappacher, and D. Wackeroth, e-print hep-ph/0108274.

[45] G. Polesello and T. Rizzo, work in progress, shown at the Workshop on Physics at TeV Colliders, Les Houches, 2001.

[46] C.D. McMullen and S. Nandi, e-print hep-ph/0110275.

[47] D. Bardin et al. (ZFITTER Collaboration), Comput. Phys. Commun. 133, 229 (2001). 
Tables 
TABLE I: Best-fit values of $\eta=\pi^{2} /\left(3 M_{C}^{2}\right)$ and the $95 \%$ C.L. upper limits on $\eta$ for individual data set and combinations. Corresponding 95\% C.L. lower limits on $M_{C}$ are also shown.

\begin{tabular}{lccc}
\hline \hline & $\eta\left(\mathrm{TeV}^{-2}\right)$ & $\eta_{95}\left(\mathrm{TeV}^{-2}\right)$ & $M_{C}^{95}(\mathrm{TeV})$ \\
\hline \hline LEP 2: & & & \\
hadronic cross section, ang. dist., $R_{b, c}$ & $-0.33_{-0.13}^{+0.13}$ & 0.12 & 5.3 \\
$\mu, \tau$ cross section \& ang. dist. & $0.09_{-0.18}^{+0.18}$ & 0.42 & 2.8 \\
ee cross section \& ang. dist. & $-0.62_{-0.20}^{+0.20}$ & 0.16 & 4.5 \\
LEP combined & $-0.28_{-0.092}^{+0.092}$ & 0.076 & 6.6 \\
\hline HERA: & $-2.74_{-1.51}^{+1.49}$ & 1.59 & 1.4 \\
NC & $-0.057_{-1.31}^{+1.28}$ & 2.45 & 1.2 \\
CC & $-1.23_{-0.99}^{+0.98}$ & 1.25 & 1.6 \\
HERA combined & & & \\
\hline TEVATRON: & $-0.87_{-1.03}^{+1.12}$ & 1.96 & 1.3 \\
Drell-yan & $0.46_{-0.58}^{+0.37}$ & 1.0 & 1.8 \\
Tevatron dijet & $-0.53_{-0.49}^{+0.51}$ & 9.2 & 0.60 \\
Tevatron top production & $-0.38_{-0.48}^{+0.52}$ & 0.65 & 2.3 \\
Tevatron combined & $-0.29_{-0.090}^{+0.090}$ & 0.071 & \\
\hline \hline All combined & & & \\
\hline \hline
\end{tabular}


TABLE II: Sensitivity to the parameter $\eta=\pi^{2} / 3 M_{C}^{2}$ in Run 1 , Run 2 of the Tevatron and at the LHC, using the dilepton channel. The corresponding 95\% C.L. lower limits on $M_{C}$ are also shown.

\begin{tabular}{|c|c|c|}
\hline & $\eta_{95}\left(\mathrm{TeV}^{-2}\right)$ & $95 \%$ C.L. lower limit on $M_{C}(\mathrm{TeV})$ \\
\hline \multicolumn{3}{|l|}{$\underline{\operatorname{Run} 1\left(120 \mathrm{pb}^{-1}\right)}$} \\
\hline Dilepton & 1.62 & 1.4 \\
\hline \multicolumn{3}{|l|}{$\underline{\operatorname{Run} 2 \mathrm{a}\left(2 \mathrm{fb}^{-1}\right)}$} \\
\hline Dilepton & 0.40 & 2.9 \\
\hline \multicolumn{3}{|l|}{$\underline{\operatorname{Run} 2 \mathrm{~b}\left(15 \mathrm{fb}^{-1}\right)}$} \\
\hline Dilepton & 0.19 & 4.2 \\
\hline \multicolumn{3}{|c|}{$\underline{\mathrm{LHC}}\left(14 \mathrm{TeV}, 100 \mathrm{fb}^{-1}, 3 \%\right.$ systematics $)$} \\
\hline Dilepton & $1.81 \times 10^{-2}$ & 13.5 \\
\hline \multicolumn{3}{|c|}{$\underline{\mathrm{LHC}}\left(14 \mathrm{TeV}, 100 \mathrm{fb}^{-1}, 1 \%\right.$ systematics $)$} \\
\hline Dilepton & $1.37 \times 10^{-2}$ & 15.5 \\
\hline
\end{tabular}


TABLE III: ZEUS: differential cross-section $d \sigma / d Q^{2}$ of the $e^{+} p \rightarrow e^{+} X$ production. The following quantities are given for each bin: the $Q^{2}$ range, the measured Born-level cross-section, and the SM prediction for the Born-level cross section.

\begin{tabular}{|c|c|c|}
\hline & \multicolumn{2}{|c|}{$d \sigma / d Q^{2}\left(\mathrm{pb} / \mathrm{GeV}^{2}\right)$} \\
\hline$\left(\mathrm{GeV}^{2}\right)$ & Measured & $\mathrm{SM}$ \\
\hline $400.0-475.7$ & $2.753 \pm 0.035_{-0.051}^{+0.066}$ & 2.673 \\
\hline $475.7-565.7$ & $1.753 \pm 0.024_{-0.039}^{+0.047}$ & 1.775 \\
\hline $565.7-672.7$ & $1.187 \pm 0.018_{-0.023}^{+0.022}$ & 1.149 \\
\hline $672.7-800.0$ & $\left(7.71 \pm 0.13_{-0.36}^{+0.14}\right) \cdot 10^{-1}$ & $7.65 \cdot 10^{-1}$ \\
\hline $800.0-951.4$ & $\left(4.79 \pm 0.09_{-0.21}^{+0.10}\right) \cdot 10^{-1}$ & $4.93 \cdot 10^{-1}$ \\
\hline $951.4-1131.4$ & $\left(3.21 \pm 0.07_{-0.06}^{+0.06}\right) \cdot 10^{-1}$ & $3.13 \cdot 10^{-1}$ \\
\hline $1131.4-1345.4$ & $\left(2.01 \pm 0.05_{-0.03}^{+0.04}\right) \cdot 10^{-1}$ & $2.04 \cdot 10^{-1}$ \\
\hline $1345.4-1600.0$ & $\left(1.27 \pm 0.033_{-0.02}^{+0.03}\right) \cdot 10^{-1}$ & $1.28 \cdot 10^{-1}$ \\
\hline $1600.0-1902.7$ & $\left(8.49 \pm 0.266_{-0.30}^{+0.17}\right) \cdot 10^{-2}$ & $8.26 \cdot 10^{-2}$ \\
\hline $1902.7-2262.8$ & $\left(4.97 \pm 0.18_{-0.16}^{+0.11}\right) \cdot 10^{-2}$ & $5.01 \cdot 10^{-2}$ \\
\hline $2262.8-2690.9$ & $\left(3.05 \pm 0.13_{-0.14}^{+0.06}\right) \cdot 10^{-2}$ & $3.13 \cdot 10^{-2}$ \\
\hline $2690.9-3200.0$ & $\left(1.99 \pm 0.10_{-0.09}^{+0.07}\right) \cdot 10^{-2}$ & $2.09 \cdot 10^{-2}$ \\
\hline $3200.0-4525.5$ & $\left(9.00 \pm 0.39_{-0.24}^{+0.20}\right) \cdot 10^{-3}$ & $9.77 \cdot 10^{-3}$ \\
\hline $4525.5-6400.0$ & $\left(3.30 \pm 0.19_{-0.10}^{+0.17}\right) \cdot 10^{-3}$ & $3.49 \cdot 10^{-3}$ \\
\hline $6400.0-9050.0$ & $\left(1.32 \pm 0.10_{-0.07}^{+0.02}\right) \cdot 10^{-3}$ & $1.20 \cdot 10^{-3}$ \\
\hline $9050.0-12800.0$ & $\left(3.69_{-0.47}^{+0.53}{ }_{-0.11}^{+0.08}\right) \cdot 10^{-4}$ & $3.64 \cdot 10^{-4}$ \\
\hline $12800.0-18102.0$ & $\left(8.9_{-2.0}^{+2.5}{ }_{-0.6}^{+0.7}\right) \cdot 10^{-5}$ & $10.0 \cdot 10^{-5}$ \\
\hline $18102.0-25600.0$ & $\left(2.4_{-0.8}^{+1.2+0.4}+0.1\right) \cdot 10^{-5}$ & $2.2 \cdot 10^{-5}$ \\
\hline $25600.0-36203.0$ & $<6.0 \cdot 10^{-6}$ & $3.7 \cdot 10^{-6}$ \\
\hline $36203.0-51200.0$ & $\left(2.6_{-1.7}^{+3.5}{ }_{-0.2}^{+0.7}\right) \cdot 10^{-6}$ & $0.4 \cdot 10^{-6}$ \\
\hline
\end{tabular}


TABLE IV: ZEUS: differential cross section $d \sigma / d Q^{2}$ of the $e^{+} p \rightarrow \bar{\nu}_{e} X$ production. The following quantities are given for each bin: the $Q^{2}$ range; the measured Born-level cross section $d \sigma / d Q^{2}$, and the SM prediction for the Born-level cross section.

\begin{tabular}{rcr}
\hline \hline $\begin{array}{c}Q^{2} \text { range } \\
\left(\mathrm{GeV}^{2}\right)\end{array}$ & $d \sigma / d Q^{2}\left(\mathrm{pb} / \mathrm{GeV}^{2}\right)$ \\
\hline $200-400$ & measured & $\mathrm{SM}$ \\
$400-711$ & $(1.82 \pm 0.14 \pm 0.08) \cdot 10^{-2}$ & $2.80 \cdot 10^{-2}$ \\
$711-1265$ & $(1.29 \pm 0.08 \pm 0.03) \cdot 10^{-2}$ & $1.87 \cdot 10^{-2}$ \\
$1265-2249$ & $\left.(5.62 \pm 0.40 \pm 0.08) \cdot 10_{-0.34}^{-3}\right) \cdot 10^{-2}$ & $1.15 \cdot 10^{-2}$ \\
$2249-4000$ & $\left(2.62 \pm 0.20_{-0.09}^{+0.04}\right) \cdot 10^{-3}$ & $6.07 \cdot 10^{-3}$ \\
$4000-7113$ & $\left(7.91_{-0.83}^{+0.93}+0.38\right) \cdot 10^{-4}$ & $2.61 \cdot 10^{-3}$ \\
$7113-12649$ & $\left(2.00_{-0.30}^{+0.35} \pm 0.17\right) \cdot 10^{-4}$ & $8.29 \cdot 10^{-4}$ \\
$12649-22494$ & $\left(2.61_{-0.72}^{+0.95}{ }_{-0.38}^{+0.45}\right) \cdot 10^{-5}$ & $1.65 \cdot 10^{-4}$ \\
$22494-60000$ & $\left(5.9_{-4.9}^{+14 .+1.8}\right) \cdot 10^{-7}$ & $1.71 \cdot 10^{-5}$ \\
\hline \hline
\end{tabular}


TABLE V: H1: reduced NC cross-section $\tilde{\sigma}_{\mathrm{NC}}\left(x, Q^{2}\right)$ in the $e^{+} p$ collisions obtained by dividing $d^{2} \sigma_{\mathrm{NC}} / d x d Q^{2}$ by the kinematic factor $x Q^{4} /\left(Y_{+} 2 \pi \alpha^{2}\right)$, with its statistical $\left(\delta_{\text {stat }}\right)$, systematic $\left(\delta_{\text {sys }}\right)$, and combined $\left(\delta_{\text {tot }}\right)$ uncertainties. The additional normalization uncertainty, not included in the systematic error, is $1.5 \%$. The table continues on the next two pages.

\begin{tabular}{|c|c|c|c|c|c|c|}
\hline $\begin{array}{c}Q^{2} \\
\left(\mathrm{GeV}^{2}\right) \\
\end{array}$ & $x$ & $y$ & $\tilde{\sigma}_{\mathrm{NC}}$ & $\begin{array}{r}\delta_{\text {stat }} \\
(\%) \\
\end{array}$ & $\begin{array}{r}\delta_{\mathrm{sys}} \\
(\%) \\
\end{array}$ & $\begin{array}{r}\delta_{\text {tot }} \\
(\%) \\
\end{array}$ \\
\hline 150 & 0.003 & 0.518 & 1.240 & 1.8 & 5.2 & 5.5 \\
\hline 150 & 0.005 & 0.331 & 1.100 & 1.8 & 3.3 & 3.8 \\
\hline 150 & 0.008 & 0.207 & 0.920 & 2.9 & 8.9 & 9.3 \\
\hline 200 & 0.005 & 0.442 & 1.102 & 1.8 & 5.0 & 5.3 \\
\hline 200 & 0.008 & 0.276 & 0.915 & 1.9 & 3.5 & 4.0 \\
\hline 200 & 0.013 & 0.170 & 0.765 & 2.2 & 3.7 & 4.3 \\
\hline 200 & 0.020 & 0.110 & 0.696 & 2.6 & 4.9 & 5.5 \\
\hline 200 & 0.032 & 0.069 & 0.601 & 3.2 & 7.5 & 8.1 \\
\hline 200 & 0.050 & 0.044 & 0.516 & 3.7 & 8.2 & 9.0 \\
\hline 200 & 0.080 & 0.028 & 0.439 & 4.2 & 9.0 & 9.9 \\
\hline 250 & 0.005 & 0.552 & 1.113 & 2.3 & 5.1 & 5.6 \\
\hline 250 & 0.008 & 0.345 & 1.018 & 2.0 & 3.7 & 4.2 \\
\hline 250 & 0.013 & 0.212 & 0.807 & 2.1 & 3.9 & 4.4 \\
\hline 250 & 0.020 & 0.138 & 0.721 & 2.1 & 3.6 & 4.1 \\
\hline 250 & 0.032 & 0.086 & 0.606 & 2.2 & 3.6 & 4.3 \\
\hline 250 & 0.050 & 0.055 & 0.529 & 2.4 & 3.4 & 4.2 \\
\hline 250 & 0.080 & 0.035 & 0.430 & 2.7 & 3.6 & 4.5 \\
\hline 250 & 0.130 & 0.021 & 0.334 & 3.4 & 4.3 & 5.5 \\
\hline 250 & 0.250 & 0.011 & 0.240 & 3.3 & 7.4 & 8.1 \\
\hline 250 & 0.400 & 0.007 & 0.122 & 5.9 & 12.1 & 13.4 \\
\hline 300 & 0.005 & 0.663 & 1.139 & 3.4 & 5.6 & 6.5 \\
\hline 300 & 0.008 & 0.414 & 0.989 & 2.4 & 5.1 & 5.7 \\
\hline 300 & 0.013 & 0.255 & 0.846 & 2.4 & 3.8 & 4.5 \\
\hline 300 & 0.020 & 0.166 & 0.740 & 2.4 & 3.9 & 4.6 \\
\hline 300 & 0.032 & 0.104 & 0.629 & 2.4 & 3.7 & 4.4 \\
\hline 300 & 0.050 & 0.066 & 0.499 & 2.6 & 3.6 & 4.5 \\
\hline 300 & 0.080 & 0.041 & 0.456 & 2.7 & 3.9 & 4.8 \\
\hline 300 & 0.130 & 0.025 & 0.346 & 3.4 & 5.8 & 6.8 \\
\hline 300 & 0.250 & 0.013 & 0.250 & 3.1 & 8.1 & 8.7 \\
\hline 300 & 0.400 & 0.008 & 0.140 & 5.7 & 14.5 & 15.6 \\
\hline 400 & 0.008 & 0.552 & 0.976 & 3.1 & 5.1 & 6.0 \\
\hline 400 & 0.013 & 0.340 & 0.841 & 2.8 & 3.9 & 4.8 \\
\hline 400 & 0.020 & 0.221 & 0.739 & 2.8 & 3.7 & 4.7 \\
\hline 400 & 0.032 & 0.138 & 0.619 & 2.8 & 3.6 & 4.6 \\
\hline 400 & 0.050 & 0.088 & 0.513 & 3.0 & 3.8 & 4.8 \\
\hline 400 & 0.080 & 0.055 & 0.455 & 3.1 & 4.0 & 5.1 \\
\hline 400 & 0.130 & 0.034 & 0.373 & 3.8 & 4.5 & 5.9 \\
\hline 400 & 0.250 & 0.018 & 0.241 & 3.5 & 6.5 & 7.4 \\
\hline 400 & 0.400 & 0.011 & 0.155 & 6.2 & 11.6 & 13.2 \\
\hline
\end{tabular}




\begin{tabular}{|c|c|c|c|c|c|c|}
\hline $\begin{array}{c}Q^{2} \\
\left(\mathrm{GeV}^{2}\right) \\
\end{array}$ & $x$ & $y$ & $\tilde{\sigma}_{\mathrm{NC}}$ & $\begin{array}{r}\delta_{\text {stat }} \\
(\%) \\
\end{array}$ & $\begin{array}{c}\delta_{\mathrm{sys}} \\
(\%)\end{array}$ & $\begin{array}{r}\delta_{\text {tot }} \\
(\%)\end{array}$ \\
\hline 500 & 0.008 & 0.690 & 1.026 & 4.2 & 5.1 & 6.6 \\
\hline 500 & 0.013 & 0.425 & 0.906 & 3.3 & 5.2 & 6.2 \\
\hline 500 & 0.020 & 0.276 & 0.792 & 3.3 & 3.9 & 5.2 \\
\hline 500 & 0.032 & 0.173 & 0.654 & 3.3 & 4.0 & 5.2 \\
\hline 500 & 0.050 & 0.110 & 0.508 & 3.5 & 4.1 & 5.4 \\
\hline 500 & 0.080 & 0.069 & 0.445 & 3.6 & 3.7 & 5.2 \\
\hline 500 & 0.130 & 0.042 & 0.368 & 4.3 & 4.3 & 6.1 \\
\hline 500 & 0.180 & 0.031 & 0.287 & 4.9 & 5.4 & 7.3 \\
\hline 500 & 0.250 & 0.022 & 0.220 & 5.9 & 8.5 & 10.4 \\
\hline 500 & 0.400 & 0.014 & 0.143 & 8.6 & 15.3 & 17.5 \\
\hline 650 & 0.013 & 0.552 & 0.903 & 4.0 & 4.3 & 5.9 \\
\hline 650 & 0.020 & 0.359 & 0.718 & 4.1 & 3.9 & 5.7 \\
\hline 650 & 0.032 & 0.224 & 0.633 & 4.0 & 4.0 & 5.7 \\
\hline 650 & 0.050 & 0.144 & 0.521 & 4.1 & 3.9 & 5.7 \\
\hline 650 & 0.080 & 0.090 & 0.436 & 4.0 & 4.0 & 5.7 \\
\hline 650 & 0.130 & 0.055 & 0.413 & 4.6 & 4.7 & 6.6 \\
\hline 650 & 0.180 & 0.040 & 0.309 & 5.3 & 5.8 & 7.9 \\
\hline 650 & 0.250 & 0.029 & 0.246 & 6.2 & 8.7 & 10.6 \\
\hline 650 & 0.400 & 0.018 & 0.125 & 9.9 & 11.5 & 15.2 \\
\hline 650 & 0.650 & 0.011 & 0.021 & 14.3 & 15.7 & 21.3 \\
\hline 800 & 0.013 & 0.680 & 1.000 & 5.0 & 4.7 & 6.8 \\
\hline 800 & 0.020 & 0.442 & 0.796 & 4.6 & 4.3 & 6.3 \\
\hline 800 & 0.032 & 0.276 & 0.709 & 4.5 & 4.0 & 6.0 \\
\hline 800 & 0.050 & 0.177 & 0.540 & 4.6 & 3.9 & 6.0 \\
\hline 800 & 0.080 & 0.110 & 0.474 & 4.6 & 4.2 & 6.2 \\
\hline 800 & 0.130 & 0.068 & 0.370 & 5.4 & 4.8 & 7.2 \\
\hline 800 & 0.180 & 0.049 & 0.333 & 6.0 & 4.9 & 7.8 \\
\hline 800 & 0.250 & 0.035 & 0.208 & 7.5 & 5.8 & 9.4 \\
\hline 800 & 0.400 & 0.022 & 0.150 & 9.6 & 10.5 & 14.2 \\
\hline 800 & 0.650 & 0.014 & 0.018 & 19.6 & 18.4 & 26.9 \\
\hline 1000 & 0.020 & 0.552 & 0.754 & 5.4 & 3.8 & 6.6 \\
\hline 1000 & 0.032 & 0.345 & 0.639 & 5.6 & 4.1 & 6.9 \\
\hline 1000 & 0.050 & 0.221 & 0.566 & 5.1 & 3.8 & 6.4 \\
\hline 1000 & 0.080 & 0.138 & 0.431 & 5.3 & 3.7 & 6.5 \\
\hline 1000 & 0.130 & 0.085 & 0.385 & 6.1 & 4.8 & 7.7 \\
\hline 1000 & 0.180 & 0.061 & 0.341 & 6.7 & 4.3 & 7.9 \\
\hline 1000 & 0.250 & 0.044 & 0.244 & 7.8 & 5.4 & 9.5 \\
\hline 1000 & 0.400 & 0.028 & 0.111 & 12.1 & 13.4 & 18.1 \\
\hline 1000 & 0.650 & 0.017 & 0.013 & 25.0 & 15.1 & 29.2 \\
\hline 1200 & 0.020 & 0.663 & 0.737 & 7.2 & 3.7 & 8.1 \\
\hline 1200 & 0.032 & 0.414 & 0.645 & 6.4 & 3.8 & 7.4 \\
\hline 1200 & 0.050 & 0.265 & 0.531 & 6.0 & 3.5 & 6.9 \\
\hline 1200 & 0.080 & 0.166 & 0.448 & 5.9 & 3.6 & 6.9 \\
\hline 1200 & 0.130 & 0.102 & 0.391 & 6.8 & 3.7 & 7.8 \\
\hline 1200 & 0.180 & 0.074 & 0.338 & 7.5 & 4.7 & 8.9 \\
\hline 1200 & 0.250 & 0.053 & 0.250 & 8.7 & 6.7 & 10.9 \\
\hline 1200 & 0.400 & 0.033 & 0.129 & 12.1 & 8.5 & 14.8 \\
\hline 1200 & 0.650 & 0.020 & 0.017 & 24.2 & 17.5 & 29.9 \\
\hline
\end{tabular}




\begin{tabular}{|c|c|c|c|c|c|c|}
\hline $\begin{array}{c}Q^{2} \\
\left(\mathrm{GeV}^{2}\right)\end{array}$ & $x$ & $y$ & $\tilde{\sigma}_{\mathrm{NC}}$ & $\begin{array}{r}\delta_{\text {stat }} \\
(\%)\end{array}$ & $\begin{array}{c}\delta_{\mathrm{sys}} \\
(\%)\end{array}$ & $\begin{array}{r}\delta_{\text {tot }} \\
(\%)\end{array}$ \\
\hline 1500 & 0.020 & 0.828 & 0.789 & 9.2 & 5.0 & 10.5 \\
\hline 1500 & 0.032 & 0.518 & 0.581 & 8.1 & 4.3 & 9.2 \\
\hline 1500 & 0.050 & 0.331 & 0.486 & 7.2 & 3.8 & 8.1 \\
\hline 1500 & 0.080 & 0.207 & 0.457 & 6.8 & 3.7 & 7.8 \\
\hline 1500 & 0.130 & 0.127 & 0.376 & 8.0 & 3.9 & 8.9 \\
\hline 1500 & 0.180 & 0.092 & 0.345 & 8.6 & 4.2 & 9.6 \\
\hline 1500 & 0.250 & 0.066 & 0.268 & 9.4 & 5.8 & 11.0 \\
\hline 1500 & 0.400 & 0.041 & 0.110 & 14.6 & 7.8 & 16.6 \\
\hline 1500 & 0.650 & 0.025 & 0.009 & 37.8 & 19.6 & 42.6 \\
\hline 2000 & 0.032 & 0.690 & 0.614 & 9.0 & 4.1 & 9.9 \\
\hline 2000 & 0.050 & 0.442 & 0.541 & 8.7 & 4.3 & 9.7 \\
\hline 2000 & 0.080 & 0.276 & 0.428 & 8.3 & 3.9 & 9.1 \\
\hline 2000 & 0.130 & 0.170 & 0.340 & 9.6 & 4.3 & 10.6 \\
\hline 2000 & 0.180 & 0.123 & 0.331 & 10.1 & 4.8 & 11.1 \\
\hline 2000 & 0.250 & 0.088 & 0.249 & 10.7 & 5.9 & 12.2 \\
\hline 2000 & 0.400 & 0.055 & 0.114 & 15.1 & 8.2 & 17.2 \\
\hline 2000 & 0.650 & 0.034 & 0.011 & 37.8 & 18.7 & 42.2 \\
\hline 3000 & 0.050 & 0.663 & 0.513 & 7.3 & 4.1 & 8.4 \\
\hline 3000 & 0.080 & 0.414 & 0.458 & 7.7 & 4.2 & 8.7 \\
\hline 3000 & 0.130 & 0.255 & 0.347 & 9.1 & 4.8 & 10.2 \\
\hline 3000 & 0.180 & 0.184 & 0.324 & 9.2 & 4.1 & 10.0 \\
\hline 3000 & 0.250 & 0.133 & 0.242 & 9.9 & 4.9 & 11.1 \\
\hline 3000 & 0.400 & 0.083 & 0.127 & 12.5 & 9.0 & 15.4 \\
\hline 3000 & 0.650 & 0.051 & 0.012 & 30.1 & 14.9 & 33.6 \\
\hline 5000 & 0.080 & 0.690 & 0.353 & 10.4 & 4.7 & 11.4 \\
\hline 5000 & 0.130 & 0.425 & 0.392 & 10.4 & 5.0 & 11.6 \\
\hline 5000 & 0.180 & 0.307 & 0.223 & 13.4 & 4.5 & 14.1 \\
\hline 5000 & 0.250 & 0.221 & 0.217 & 13.9 & 6.6 & 15.4 \\
\hline 5000 & 0.400 & 0.138 & 0.127 & 17.1 & 8.8 & 19.3 \\
\hline 5000 & 0.650 & 0.085 & 0.012 & 37.8 & 14.9 & 40.6 \\
\hline 8000 & 0.130 & 0.680 & 0.283 & 16.5 & 4.9 & 17.2 \\
\hline 8000 & 0.180 & 0.491 & 0.284 & 15.5 & 6.4 & 16.7 \\
\hline 8000 & 0.250 & 0.353 & 0.273 & 15.1 & 7.0 & 16.6 \\
\hline 8000 & 0.400 & 0.221 & 0.093 & 24.2 & 9.9 & 26.2 \\
\hline 8000 & 0.650 & 0.136 & 0.013 & 44.7 & 19.8 & 48.9 \\
\hline 12000 & 0.180 & 0.736 & 0.153 & 34.4 & 4.3 & 34.6 \\
\hline 12000 & 0.250 & 0.530 & 0.127 & 32.1 & 6.2 & 32.7 \\
\hline 12000 & 0.400 & 0.331 & 0.085 & 33.3 & 11.4 & 35.2 \\
\hline 12000 & 0.650 & 0.204 & 0.015 & 57.7 & 24.2 & 62.6 \\
\hline 20000 & 0.250 & 0.884 & 0.090 & 61.9 & 5.5 & 62.2 \\
\hline 20000 & 0.400 & 0.552 & 0.142 & 35.7 & 9.9 & 37.0 \\
\hline 20000 & 0.650 & 0.340 & 0.021 & 70.7 & 41.6 & 82.0 \\
\hline 30000 & 0.400 & 0.828 & 0.182 & 71.9 & 9.6 & 72.6 \\
\hline
\end{tabular}


TABLE VI: H1: double differential CC cross-section $d^{2} \sigma_{\mathrm{CC}} / d x d Q^{2}$ with its statistical $\left(\delta_{\text {stat }}\right)$, systematic $\left(\delta_{\text {sys }}\right)$, and combined $\left(\delta_{\text {tot }}\right)$ uncertainties in the $e^{+} p$ collisions. The additional normalization uncertainty, not included in the systematic error, is $1.5 \%$.

\begin{tabular}{|c|c|c|c|c|c|c|}
\hline $\begin{array}{c}Q^{2} \\
\left(\mathrm{GeV}^{2}\right)\end{array}$ & $x$ & $y$ & $\begin{array}{r}d^{2} \sigma_{\mathrm{CC}} / d x d Q^{2} \\
\left(\mathrm{pb} / \mathrm{GeV}^{2}\right)\end{array}$ & $\begin{array}{r}\delta_{\text {stat }} \\
(\%)\end{array}$ & $\begin{array}{l}\delta_{\text {sys }} \\
(\%)\end{array}$ & $\begin{array}{l}\delta_{\text {tot }} \\
(\%)\end{array}$ \\
\hline 300 & 0.013 & 0.255 & $0.637 \cdot 10^{0}$ & 27.4 & 16.0 & 31.8 \\
\hline 300 & 0.032 & 0.104 & $0.124 \cdot 10^{0}$ & 28.1 & 10.3 & 30.0 \\
\hline 300 & 0.080 & 0.041 & $0.532 \cdot 10^{-1}$ & 23.8 & 7.5 & 25.5 \\
\hline 500 & 0.013 & 0.425 & $0.468 \cdot 10^{0}$ & 25.1 & 15.7 & 29.7 \\
\hline 500 & 0.032 & 0.173 & $0.177 \cdot 10^{0}$ & 17.0 & 8.7 & 19.2 \\
\hline 500 & 0.080 & 0.069 & $0.546 \cdot 10^{-1}$ & 17.0 & 6.5 & 18.9 \\
\hline 500 & 0.130 & 0.043 & $0.289 \cdot 10^{-1}$ & 27.8 & 8.0 & 29.4 \\
\hline 1000 & 0.032 & 0.345 & $0.124 \cdot 10^{0}$ & 15.0 & 8.0 & 17.1 \\
\hline 1000 & 0.080 & 0.138 & $0.487 \cdot 10^{-1}$ & 13.3 & 6.1 & 14.8 \\
\hline 1000 & 0.130 & 0.085 & $0.199 \cdot 10^{-1}$ & 20.9 & 6.5 & 22.5 \\
\hline 1000 & 0.250 & 0.044 & $0.105 \cdot 10^{-1}$ & 31.7 & 11.7 & 34.1 \\
\hline 2000 & 0.032 & 0.690 & $0.716 \cdot 10^{-1}$ & 15.7 & 8.8 & 18.1 \\
\hline 2000 & 0.080 & 0.276 & $0.264 \cdot 10^{-1}$ & 13.5 & 5.8 & 14.8 \\
\hline 2000 & 0.130 & 0.170 & $0.949 \cdot 10^{-2}$ & 20.6 & 5.7 & 21.4 \\
\hline 2000 & 0.250 & 0.088 & $0.566 \cdot 10^{-2}$ & 23.0 & 7.3 & 24.6 \\
\hline 3000 & 0.080 & 0.414 & $0.156 \cdot 10^{-1}$ & 15.2 & 6.7 & 16.8 \\
\hline 3000 & 0.130 & 0.255 & $0.872 \cdot 10^{-2}$ & 17.0 & 5.9 & 18.1 \\
\hline 3000 & 0.250 & 0.133 & $0.283 \cdot 10^{-2}$ & 23.6 & 8.2 & 25.1 \\
\hline 5000 & 0.130 & 0.425 & $0.402 \cdot 10^{-2}$ & 21.0 & 7.4 & 22.3 \\
\hline 5000 & 0.250 & 0.221 & $0.111 \cdot 10^{-2}$ & 26.8 & 6.5 & 27.6 \\
\hline 8000 & 0.130 & 0.680 & $0.125 \cdot 10^{-2}$ & 35.7 & 14.3 & 38.5 \\
\hline 8000 & 0.250 & 0.354 & $0.530 \cdot 10^{-3}$ & 33.5 & 11.2 & 35.4 \\
\hline 8000 & 0.400 & 0.221 & $0.235 \cdot 10^{-3}$ & 50.0 & 15.6 & 52.4 \\
\hline 15000 & 0.250 & 0.663 & $0.774 \cdot 10^{-4}$ & 71.2 & 18.1 & 73.5 \\
\hline 15000 & 0.400 & 0.414 & $0.114 \cdot 10^{-3}$ & 40.9 & 17.4 & 44.5 \\
\hline
\end{tabular}


TABLE VII: H1: reduced NC cross section $\tilde{\sigma}_{\mathrm{NC}}\left(x, Q^{2}\right)$ with its combined $\left(\delta_{\text {tot }}\right)$, statistical $\left(\delta_{\text {stat }}\right)$, and systematic $\left(\delta_{\mathrm{sys}}\right)$ uncertainties in the $e^{-} p$ collisions. The additional normalization uncertainty of $1.8 \%$ is not included in the errors. The table continues on the next two pages.

\begin{tabular}{|c|c|c|c|c|c|}
\hline $\begin{array}{c}Q^{2} \\
\left(\mathrm{GeV}^{2}\right) \\
\end{array}$ & $x$ & $\tilde{\sigma}_{\mathrm{NC}}$ & $\begin{array}{r}\delta_{\text {tot }} \\
(\%) \\
\end{array}$ & $\begin{array}{r}\delta_{\text {stat }} \\
(\%) \\
\end{array}$ & $\begin{array}{r}\delta_{\mathrm{sys}} \\
(\%) \\
\end{array}$ \\
\hline 150 & 0.0032 & 1.218 & 4.7 & 2.7 & 3.8 \\
\hline 150 & 0.0050 & 1.154 & 4.4 & 2.8 & 3.4 \\
\hline 150 & 0.0080 & 0.968 & 9.1 & 4.1 & 8.2 \\
\hline 200 & 0.0032 & 1.271 & 6.1 & 4.1 & 4.5 \\
\hline 200 & 0.0050 & 1.107 & 4.6 & 2.8 & 3.6 \\
\hline 200 & 0.0080 & 0.915 & 4.5 & 3.0 & 3.3 \\
\hline 200 & 0.0130 & 0.860 & 4.7 & 3.2 & 3.5 \\
\hline 200 & 0.0200 & 0.677 & 6.5 & 3.8 & 5.3 \\
\hline 200 & 0.0320 & 0.558 & 8.6 & 4.5 & 7.4 \\
\hline 200 & 0.0500 & 0.506 & 9.9 & 5.2 & 8.4 \\
\hline 200 & 0.0800 & 0.407 & 12.4 & 5.9 & 10.9 \\
\hline 250 & 0.0050 & 1.123 & 5.3 & 3.5 & 4.0 \\
\hline 250 & 0.0080 & 1.021 & 5.3 & 3.2 & 4.2 \\
\hline 250 & 0.0130 & 0.825 & 5.7 & 3.4 & 4.5 \\
\hline 250 & 0.0200 & 0.691 & 5.4 & 3.5 & 4.0 \\
\hline 250 & 0.0320 & 0.569 & 6.1 & 3.8 & 4.7 \\
\hline 250 & 0.0500 & 0.493 & 5.7 & 4.3 & 3.7 \\
\hline 250 & 0.0800 & 0.407 & 6.1 & 4.7 & 3.9 \\
\hline 250 & 0.1300 & 0.311 & 7.8 & 5.3 & 5.8 \\
\hline 250 & 0.2500 & 0.225 & 12.1 & 7.5 & 9.5 \\
\hline 250 & 0.4000 & 0.138 & 11.8 & 9.3 & 7.2 \\
\hline 300 & 0.0050 & 1.152 & 7.2 & 5.6 & 4.6 \\
\hline 300 & 0.0080 & 1.026 & 5.1 & 3.6 & 3.6 \\
\hline 300 & 0.0130 & 0.878 & 5.3 & 3.8 & 3.7 \\
\hline 300 & 0.0200 & 0.735 & 5.9 & 4.0 & 4.3 \\
\hline 300 & 0.0320 & 0.605 & 5.8 & 4.2 & 4.1 \\
\hline 300 & 0.0500 & 0.509 & 6.8 & 4.5 & 5.1 \\
\hline 300 & 0.0800 & 0.390 & 6.9 & 5.2 & 4.6 \\
\hline 300 & 0.1300 & 0.332 & 8.8 & 5.4 & 7.0 \\
\hline 300 & 0.2500 & 0.277 & 12.8 & 6.9 & 10.8 \\
\hline 300 & 0.4000 & 0.143 & 14.2 & 10.3 & 9.8 \\
\hline 400 & 0.0080 & 1.088 & 6.1 & 4.5 & 4.1 \\
\hline 400 & 0.0130 & 0.897 & 5.6 & 4.3 & 3.6 \\
\hline 400 & 0.0200 & 0.732 & 5.8 & 4.5 & 3.6 \\
\hline 400 & 0.0320 & 0.560 & 6.1 & 4.8 & 3.8 \\
\hline 400 & 0.0500 & 0.514 & 6.3 & 5.0 & 3.7 \\
\hline 400 & 0.0800 & 0.429 & 7.0 & 5.5 & 4.3 \\
\hline 400 & 0.1300 & 0.352 & 7.5 & 5.6 & 5.0 \\
\hline 400 & 0.2500 & 0.240 & 10.6 & 7.6 & 7.4 \\
\hline 400 & 0.4000 & 0.143 & 13.7 & 10.8 & 8.4 \\
\hline
\end{tabular}




\begin{tabular}{|c|c|c|c|c|c|}
\hline $\begin{array}{c}Q^{2} \\
\left(\mathrm{GeV}^{2}\right) \\
\end{array}$ & $x$ & $\tilde{\sigma}_{\mathrm{NC}}$ & $\begin{array}{r}\delta_{\text {tot }} \\
(\%)\end{array}$ & $\begin{array}{r}\delta_{\text {stat }} \\
(\%)\end{array}$ & $\begin{array}{r}\delta_{\text {sys }} \\
(\%)\end{array}$ \\
\hline 500 & 0.0080 & 1.044 & 9.3 & 7.8 & 5.1 \\
\hline 500 & 0.0130 & 1.003 & 6.8 & 5.1 & 4.5 \\
\hline 500 & 0.0200 & 0.765 & 7.0 & 5.1 & 4.8 \\
\hline 500 & 0.0320 & 0.604 & 7.0 & 5.3 & 4.5 \\
\hline 500 & 0.0500 & 0.517 & 6.9 & 5.6 & 4.0 \\
\hline 500 & 0.0800 & 0.392 & 9.2 & 6.4 & 6.5 \\
\hline 500 & 0.1300 & 0.363 & 8.7 & 7.2 & 4.9 \\
\hline 500 & 0.1800 & 0.283 & 11.5 & 8.2 & 8.1 \\
\hline 500 & 0.2500 & 0.254 & 14.2 & 10.5 & 9.5 \\
\hline 500 & 0.4000 & 0.139 & 21.6 & 15.4 & 15.1 \\
\hline 500 & 0.6500 & 0.026 & 22.4 & 19.6 & 10.9 \\
\hline 650 & 0.0130 & 0.988 & 7.3 & 6.0 & 4.1 \\
\hline 650 & 0.0200 & 0.791 & 7.7 & 6.3 & 4.4 \\
\hline 650 & 0.0320 & 0.684 & 7.4 & 6.1 & 4.3 \\
\hline 650 & 0.0500 & 0.538 & 8.3 & 6.5 & 5.2 \\
\hline 650 & 0.0800 & 0.436 & 9.2 & 7.1 & 5.8 \\
\hline 650 & 0.1300 & 0.343 & 10.5 & 8.8 & 5.8 \\
\hline 650 & 0.1800 & 0.330 & 11.8 & 9.1 & 7.5 \\
\hline 650 & 0.2500 & 0.251 & 15.9 & 11.9 & 10.6 \\
\hline 650 & 0.4000 & 0.090 & 24.9 & 22.9 & 9.6 \\
\hline 800 & 0.0130 & 0.842 & 11.7 & 10.2 & 5.8 \\
\hline 800 & 0.0200 & 0.806 & 8.8 & 7.2 & 4.9 \\
\hline 800 & 0.0320 & 0.721 & 8.7 & 7.1 & 5.0 \\
\hline 800 & 0.0500 & 0.587 & 8.6 & 7.4 & 4.4 \\
\hline 800 & 0.0800 & 0.518 & 9.4 & 7.8 & 5.2 \\
\hline 800 & 0.1300 & 0.411 & 11.8 & 10.0 & 6.2 \\
\hline 800 & 0.1800 & 0.302 & 13.4 & 11.6 & 6.7 \\
\hline 800 & 0.2500 & 0.212 & 16.4 & 14.1 & 8.2 \\
\hline 800 & 0.4000 & 0.117 & 24.4 & 20.9 & 12.9 \\
\hline 800 & 0.6500 & 0.015 & 26.5 & 21.8 & 14.9 \\
\hline 1000 & 0.0130 & 0.773 & 13.5 & 11.5 & 6.9 \\
\hline 1000 & 0.0200 & 0.787 & 9.2 & 7.9 & 4.7 \\
\hline 1000 & 0.0320 & 0.572 & 10.0 & 9.0 & 4.4 \\
\hline 1000 & 0.0500 & 0.577 & 9.5 & 8.4 & 4.5 \\
\hline 1000 & 0.0800 & 0.450 & 10.8 & 9.3 & 5.6 \\
\hline 1000 & 0.1300 & 0.491 & 11.6 & 10.3 & 5.3 \\
\hline 1000 & 0.1800 & 0.249 & 14.6 & 13.5 & 5.7 \\
\hline 1000 & 0.2500 & 0.311 & 15.9 & 13.0 & 9.2 \\
\hline 1000 & 0.4000 & 0.122 & 26.9 & 22.9 & 14.0 \\
\hline 1200 & 0.0200 & 0.839 & 10.0 & 9.1 & 4.0 \\
\hline 1200 & 0.0320 & 0.719 & 9.9 & 9.2 & 3.7 \\
\hline 1200 & 0.0500 & 0.645 & 9.9 & 9.3 & 3.6 \\
\hline 1200 & 0.0800 & 0.415 & 11.2 & 10.7 & 3.4 \\
\hline 1200 & 0.1300 & 0.384 & 13.4 & 12.6 & 4.5 \\
\hline 1200 & 0.1800 & 0.341 & 14.6 & 13.6 & 5.3 \\
\hline 1200 & 0.2500 & 0.251 & 17.3 & 15.8 & 7.0 \\
\hline 1200 & 0.4000 & 0.110 & 27.7 & 25.0 & 12.0 \\
\hline
\end{tabular}




\begin{tabular}{|c|c|c|c|c|c|}
\hline $\begin{array}{c}Q^{2} \\
\left(\mathrm{GeV}^{2}\right)\end{array}$ & $x$ & $\tilde{\sigma}_{\mathrm{NC}}$ & $\begin{array}{l}\delta_{\text {tot }} \\
(\%)\end{array}$ & $\begin{array}{r}\delta_{\text {stat }} \\
(\%)\end{array}$ & $\begin{array}{l}\delta_{\mathrm{sys}} \\
(\%)\end{array}$ \\
\hline 1500 & 0.0200 & 0.860 & 13.5 & 12.4 & 5.5 \\
\hline 1500 & 0.0320 & 0.704 & 11.4 & 10.4 & 4.7 \\
\hline 1500 & 0.0500 & 0.515 & 12.2 & 11.7 & 3.6 \\
\hline 1500 & 0.0800 & 0.512 & 11.7 & 11.0 & 4.0 \\
\hline 1500 & 0.1300 & 0.390 & 14.8 & 13.9 & 5.0 \\
\hline 1500 & 0.1800 & 0.260 & 19.1 & 18.6 & 4.3 \\
\hline 1500 & 0.2500 & 0.197 & 21.1 & 19.6 & 7.7 \\
\hline 1500 & 0.4000 & 0.145 & 27.4 & 24.3 & 12.8 \\
\hline 1500 & 0.6500 & 0.014 & 38.9 & 35.4 & 16.1 \\
\hline 2000 & 0.0320 & 0.796 & 11.9 & 11.1 & 4.4 \\
\hline 2000 & 0.0500 & 0.599 & 13.9 & 13.0 & 5.0 \\
\hline 2000 & 0.0800 & 0.582 & 13.0 & 12.3 & 4.3 \\
\hline 2000 & 0.1300 & 0.224 & 20.6 & 20.0 & 4.6 \\
\hline 2000 & 0.1800 & 0.249 & 22.7 & 21.9 & 6.3 \\
\hline 2000 & 0.2500 & 0.197 & 23.4 & 22.4 & 6.8 \\
\hline 2000 & 0.4000 & 0.108 & 29.5 & 27.7 & 10.1 \\
\hline 3000 & 0.0500 & 0.606 & 12.4 & 10.6 & 6.4 \\
\hline 3000 & 0.0800 & 0.556 & 11.8 & 10.9 & 4.5 \\
\hline 3000 & 0.1300 & 0.464 & 13.0 & 12.4 & 4.0 \\
\hline 3000 & 0.1800 & 0.347 & 16.1 & 15.3 & 5.1 \\
\hline 3000 & 0.2500 & 0.255 & 19.1 & 17.8 & 7.0 \\
\hline 3000 & 0.4000 & 0.128 & 25.5 & 23.0 & 10.9 \\
\hline 5000 & 0.0800 & 0.707 & 11.7 & 10.6 & 4.8 \\
\hline 5000 & 0.1300 & 0.536 & 14.2 & 13.1 & 5.3 \\
\hline 5000 & 0.1800 & 0.442 & 14.9 & 14.0 & 5.2 \\
\hline 5000 & 0.2500 & 0.361 & 20.3 & 17.4 & 10.5 \\
\hline 5000 & 0.4000 & 0.091 & 33.5 & 31.6 & 11.1 \\
\hline 5000 & 0.6500 & 0.010 & 45.1 & 41.0 & 18.8 \\
\hline 8000 & 0.1300 & 0.722 & 17.2 & 16.0 & 6.5 \\
\hline 8000 & 0.1800 & 0.386 & 21.2 & 20.4 & 5.8 \\
\hline 8000 & 0.2500 & 0.295 & 23.3 & 21.8 & 8.2 \\
\hline 8000 & 0.4000 & 0.197 & 32.4 & 27.7 & 16.8 \\
\hline 12000 & 0.1800 & 0.471 & 28.8 & 27.8 & 7.6 \\
\hline 12000 & 0.2500 & 0.298 & 30.2 & 28.9 & 8.6 \\
\hline 12000 & 0.4000 & 0.083 & 53.7 & 50.0 & 19.6 \\
\hline 20000 & 0.2500 & 0.349 & 52.2 & 51.1 & 10.8 \\
\hline 20000 & 0.4000 & 0.182 & 46.7 & 44.7 & 13.3 \\
\hline 20000 & 0.6500 & 0.014 & 79.8 & 70.7 & 36.9 \\
\hline 30000 & 0.4000 & 0.268 & 72.9 & 70.7 & 17.5 \\
\hline
\end{tabular}


TABLE VIII: H1: double differential CC cross section $d^{2} \sigma_{\mathrm{CC}} / d x d Q^{2}$ with its overall $\left(\delta_{\text {tot }}\right)$, statistical $\left(\delta_{\text {stat }}\right)$, and systematic uncertainties $\left(\delta_{\text {sys }}\right)$ in the $e^{-} p$ collisions. The additional normalization uncertainty of $1.8 \%$ is not included in the errors.

\begin{tabular}{|c|c|c|c|c|c|}
\hline $\begin{array}{c}Q^{2} \\
\left(\mathrm{GeV}^{2}\right)\end{array}$ & $x$ & $\begin{array}{r}d^{2} \sigma_{\mathrm{CC}} / d x d Q^{2} \\
\left(\mathrm{pb} / \mathrm{GeV}^{2}\right)\end{array}$ & $\begin{array}{l}\delta_{\text {tot }} \\
(\%)\end{array}$ & $\begin{array}{r}\delta_{\text {stat }} \\
(\%)\end{array}$ & $\begin{array}{l}\delta_{\mathrm{sys}} \\
(\%)\end{array}$ \\
\hline 300 & 0.013 & $0.458 \cdot 10^{0}$ & 57.6 & 55.4 & 15.7 \\
\hline 300 & 0.032 & $0.399 \cdot 10^{0}$ & 27.3 & 24.5 & 12.0 \\
\hline 300 & 0.080 & $0.690 \cdot 10^{-1}$ & 42.3 & 40.7 & 11.6 \\
\hline 500 & 0.013 & $0.433 \cdot 10^{0}$ & 39.9 & 37.6 & 13.3 \\
\hline 500 & 0.032 & $0.285 \cdot 10^{0}$ & 21.0 & 19.6 & 7.8 \\
\hline 500 & 0.080 & $0.790 \cdot 10^{-1}$ & 22.4 & 21.8 & 5.1 \\
\hline 500 & 0.130 & $0.551 \cdot 10^{-1}$ & 29.9 & 29.0 & 7.0 \\
\hline 1000 & 0.032 & $0.186 \cdot 10^{0}$ & 18.2 & 17.5 & 4.9 \\
\hline 1000 & 0.080 & $0.556 \cdot 10^{-1}$ & 18.4 & 17.9 & 4.3 \\
\hline 1000 & 0.130 & $0.310 \cdot 10^{-1}$ & 24.5 & 24.0 & 4.6 \\
\hline 1000 & 0.250 & $0.139 \cdot 10^{-1}$ & 39.1 & 37.6 & 10.6 \\
\hline 2000 & 0.032 & $0.132 \cdot 10^{0}$ & 16.2 & 15.5 & 4.9 \\
\hline 2000 & 0.080 & $0.571 \cdot 10^{-1}$ & 13.6 & 13.0 & 3.9 \\
\hline 2000 & 0.130 & $0.197 \cdot 10^{-1}$ & 21.7 & 21.2 & 4.5 \\
\hline 2000 & 0.250 & $0.855 \cdot 10^{-2}$ & 26.4 & 25.6 & 6.5 \\
\hline 3000 & 0.080 & $0.324 \cdot 10^{-1}$ & 14.8 & 14.0 & 4.8 \\
\hline 3000 & 0.130 & $0.250 \cdot 10^{-1}$ & 15.2 & 14.0 & 6.1 \\
\hline 3000 & 0.250 & $0.749 \cdot 10^{-2}$ & 20.1 & 18.9 & 7.0 \\
\hline 3000 & 0.400 & $0.251 \cdot 10^{-2}$ & 40.3 & 35.2 & 19.6 \\
\hline 5000 & 0.080 & $0.213 \cdot 10^{-1}$ & 19.2 & 17.9 & 6.7 \\
\hline 5000 & 0.130 & $0.108 \cdot 10^{-1}$ & 18.2 & 16.8 & 7.0 \\
\hline 5000 & 0.250 & $0.550 \cdot 10^{-2}$ & 16.9 & 16.3 & 4.4 \\
\hline 5000 & 0.400 & $0.123 \cdot 10^{-2}$ & 35.6 & 33.1 & 13.1 \\
\hline 8000 & 0.130 & $0.722 \cdot 10^{-2}$ & 21.1 & 18.9 & 9.3 \\
\hline 8000 & 0.250 & $0.342 \cdot 10^{-2}$ & 17.4 & 16.3 & 6.2 \\
\hline 8000 & 0.400 & $0.946 \cdot 10^{-3}$ & 30.4 & 28.6 & 10.3 \\
\hline 15000 & 0.250 & $0.139 \cdot 10^{-2}$ & 27.3 & 22.1 & 16.0 \\
\hline 15000 & 0.400 & $0.419 \cdot 10^{-3}$ & 29.5 & 27.5 & 10.7 \\
\hline
\end{tabular}


TABLE IX: CDF: Drell-Yan data in the muon and electron channels.

\begin{tabular}{lc}
\hline \hline & Muon channel \\
Mass bin & $d^{2} \sigma /\left.d M d y\right|_{|y|<1}(\mathrm{pb} / \mathrm{GeV})$ \\
\hline $40-50$ & $0.367 \pm 0.057$ \\
$50-60$ & $0.129 \pm 0.030$ \\
$60-70$ & $0.107 \pm 0.019$ \\
$70-78$ & $0.124 \pm 0.019$ \\
$78-86$ & $0.360 \pm 0.037$ \\
$86-90$ & $2.36 \pm 0.21$ \\
$90-94$ & $12.38 \pm 1.08$ \\
$94-102$ & $0.550 \pm 0.052$ \\
$102-110$ & $0.161 \pm 0.025$ \\
$110-120$ & $0.069 \pm 0.016$ \\
$120-150$ & $0.024 \pm 0.005$ \\
$150-200$ & $0.0047 \pm 0.0016$ \\
$200-250$ & $0.0021 \pm 0.0011$ \\
$300-400$ & -300 \\
$400-500$ & $0.00107 \pm 0.00076$ \\
\hline \hline
\end{tabular}

Electron channel

\begin{tabular}{ccc} 
Mass bin & $M_{\text {mean }}(\mathrm{GeV})$ & $d \sigma / d M(\mathrm{pb} / \mathrm{GeV})$ \\
\hline \hline $40-50$ & 44.5 & $(2.300 \pm 0.448 \pm 0.126) \times 10^{0}$ \\
$50-60$ & 54.6 & $(8.038 \pm 0.978 \pm 0.478) \times 10^{-1}$ \\
$60-70$ & 63.8 & $(6.005 \pm 0.547 \pm 0.293) \times 10^{-1}$ \\
$70-78$ & 74.7 & $(5.829 \pm 0.439 \pm 0.148) \times 10^{-1}$ \\
$78-86$ & 82.9 & $(1.638 \pm 0.061 \pm 0.011) \times 10^{0}$ \\
$86-88$ & 87.1 & $(5.895 \pm 0.222 \pm 0.027) \times 10^{0}$ \\
$88-90$ & 89.2 & $(1.828 \pm 0.051 \pm 0.009) \times 10^{1}$ \\
$90-92$ & 90.6 & $(5.485 \pm 0.135 \pm 0.033) \times 10^{1}$ \\
$92-94$ & 92.8 & $(2.275 \pm 0.065 \pm 0.015) \times 10^{1}$ \\
$94-100$ & 96.2 & $(3.796 \pm 0.117 \pm 0.019) \times 10^{0}$ \\
$100-105$ & 102.2 & $(9.120 \pm 0.733 \pm 0.126) \times 10^{-1}$ \\
$105-120$ & 111.2 & $(2.631 \pm 0.237 \pm 0.044) \times 10^{-1}$ \\
$120-140$ & 128.8 & $(6.554 \pm 1.017 \pm 0.191) \times 10^{-2}$ \\
$140-200$ & 164.2 & $(2.083 \pm 0.320 \pm 0.051) \times 10^{-2}$ \\
$200-300$ & 240.6 & $(2.599 \pm 0.847 \pm 0.039) \times 10^{-3}$ \\
$300-400$ & 342.2 & $(8.080 \pm 4.677 \pm 0.127) \times 10^{-4}$ \\
$400-600$ & 478.8 & $(1.433 \pm 1.433 \pm 0.037) \times 10^{-4}$ \\
$>600$ & 725.6 & $(0.000 \pm 0.964 \pm 0.000) \times 10^{-4}$ \\
\hline \hline
\end{tabular}


TABLE X: DØ: Drell-Yan data in the electron channel.

\begin{tabular}{cc}
\hline \hline & Electron channel \\
$M_{\ell \ell}$ range $(\mathrm{GeV})$ & $\sigma(\mathrm{pb})$ \\
\hline $120-160$ & $1.93{ }_{-0.44}^{+0.43}$ \\
$160-200$ & $0.49{ }_{-0.18}^{+0.16}$ \\
$200-240$ & $0.28{ }_{-0.10}^{+0.09}$ \\
$240-290$ & $0.066{ }_{-0.058}^{+0.052}$ \\
$290-340$ & $0.033{ }_{-0.030}^{+0.032}$ \\
$340-400$ & $0.057{ }_{-0.047}^{+0.042}$ \\
$400-500$ & $<0.063(0.039)$ \\
$500-600$ & $<0.060(0.037)$ \\
$600-1000$ & $<0.058(0.035)$ \\
\hline \hline
\end{tabular}


TABLE XI: Preliminary combined LEP results on the $e^{-} e^{+} \rightarrow f \bar{f}$ production at $\sqrt{s}=130-202$ GeV. The Standard Model predictions are from ZFITTER [47] v6.10.

\begin{tabular}{|c|c|c|c|}
\hline$\sqrt{s}(\mathrm{GeV})$ & Quantity & Value & SM \\
\hline \multirow[t]{5}{*}{130} & $\sigma(q \bar{q}) \quad[\mathrm{pb}]$ & $81.938 \pm 2.220$ & 82.803 \\
\hline & $\sigma\left(\mu^{+} \mu^{-}\right)[\mathrm{pb}]$ & $8.592 \pm 0.682$ & 8.439 \\
\hline & $\sigma\left(\tau^{+} \tau^{-}\right)[\mathrm{pb}]$ & $9.082 \pm 0.931$ & 8.435 \\
\hline & $\mathrm{A}_{\mathrm{fb}}\left(\mu^{+} \mu^{-}\right)$ & $0.692 \pm 0.060$ & 0.705 \\
\hline & $\mathrm{A}_{\mathrm{fb}}\left(\tau^{+} \tau^{-}\right)$ & $0.663 \pm 0.076$ & 0.704 \\
\hline \multirow[t]{5}{*}{136} & $\sigma(q \bar{q}) \quad[\mathrm{pb}]$ & $66.570 \pm 1.967$ & 66.596 \\
\hline & $\sigma\left(\mu^{+} \mu^{-}\right)[\mathrm{pb}]$ & $8.231 \pm 0.678$ & 7.281 \\
\hline & $\sigma\left(\tau^{+} \tau^{-}\right)[\mathrm{pb}]$ & $7.123 \pm 0.821$ & 7.279 \\
\hline & $\mathrm{A}_{\mathrm{fb}}\left(\mu^{+} \mu^{-}\right)$ & $0.704 \pm 0.060$ & 0.684 \\
\hline & $\mathrm{A}_{\mathrm{fb}}\left(\tau^{+} \tau^{-}\right)$ & $0.752 \pm 0.088$ & 0.683 \\
\hline \multirow[t]{5}{*}{161} & $\sigma(q \bar{q}) \quad[\mathrm{pb}]$ & $36.909 \pm 1.071$ & 35.247 \\
\hline & $\sigma\left(\mu^{+} \mu^{-}\right)[\mathrm{pb}]$ & $4.586 \pm 0.364$ & 4.613 \\
\hline & $\sigma\left(\tau^{+} \tau^{-}\right)[\mathrm{pb}]$ & $5.692 \pm 0.545$ & 4.613 \\
\hline & $\mathrm{A}_{\mathrm{fb}}\left(\mu^{+} \mu^{-}\right)$ & $0.535 \pm 0.067$ & 0.609 \\
\hline & $\mathrm{A}_{\mathrm{fb}}\left(\tau^{+} \tau^{-}\right)$ & $0.646 \pm 0.077$ & 0.609 \\
\hline \multirow[t]{5}{*}{172} & $\sigma(q \bar{q}) \quad[\mathrm{pb}]$ & $29.172 \pm 0.987$ & 28.738 \\
\hline & $\sigma\left(\mu^{+} \mu^{-}\right)[\mathrm{pb}]$ & $3.556 \pm 0.317$ & 3.952 \\
\hline & $\sigma\left(\tau^{+} \tau^{-}\right)[\mathrm{pb}]$ & $4.026 \pm 0.450$ & 3.951 \\
\hline & $\mathrm{A}_{\mathrm{fb}}\left(\mu^{+} \mu^{-}\right)$ & $0.672 \pm 0.077$ & 0.591 \\
\hline & $\mathrm{A}_{\mathrm{fb}}\left(\tau^{+} \tau^{-}\right)$ & $0.342 \pm 0.094$ & 0.591 \\
\hline \multirow[t]{5}{*}{183} & $\sigma(q \bar{q}) \quad[\mathrm{pb}]$ & $24.567 \pm 0.421$ & 24.200 \\
\hline & $\sigma\left(\mu^{+} \mu^{-}\right)[\mathrm{pb}]$ & $3.484 \pm 0.147$ & 3.446 \\
\hline & $\sigma\left(\tau^{+} \tau^{-}\right)[\mathrm{pb}]$ & $3.398 \pm 0.174$ & 3.446 \\
\hline & $\mathrm{A}_{\mathrm{fb}}\left(\mu^{+} \mu^{-}\right)$ & $0.558 \pm 0.035$ & 0.576 \\
\hline & $\mathrm{A}_{\mathrm{fb}}\left(\tau^{+} \tau^{-}\right)$ & $0.608 \pm 0.045$ & 0.576 \\
\hline \multirow[t]{5}{*}{189} & $\begin{array}{ll}\sigma(q \bar{q}) & {[\mathrm{pb}]}\end{array}$ & $22.420 \pm 0.248$ & 22.156 \\
\hline & $\sigma\left(\mu^{+} \mu^{-}\right)[\mathrm{pb}]$ & $3.109 \pm 0.077$ & 3.207 \\
\hline & $\sigma\left(\tau^{+} \tau^{-}\right)[\mathrm{pb}]$ & $3.140 \pm 0.100$ & 3.207 \\
\hline & $\mathrm{A}_{\mathrm{fb}}\left(\mu^{+} \mu^{-}\right)$ & $0.565 \pm 0.021$ & 0.569 \\
\hline & $\mathrm{A}_{\mathrm{fb}}\left(\tau^{+} \tau^{-}\right)$ & $0.584 \pm 0.028$ & 0.569 \\
\hline \multirow[t]{5}{*}{192} & $\sigma(q \bar{q})[\mathrm{pb}]$ & $22.292 \pm 0.514$ & 21.237 \\
\hline & $\sigma\left(\mu^{+} \mu^{-}\right)[\mathrm{pb}]$ & $2.941 \pm 0.175$ & 3.097 \\
\hline & $\sigma\left(\tau^{+} \tau^{-}\right)[\mathrm{pb}]$ & $2.863 \pm 0.216$ & 3.097 \\
\hline & $\mathrm{A}_{\mathrm{fb}}\left(\mu^{+} \mu^{-}\right)$ & $0.540 \pm 0.052$ & 0.566 \\
\hline & $\mathrm{A}_{\mathrm{fb}}\left(\tau^{+} \tau^{-}\right)$ & $0.610 \pm 0.071$ & 0.566 \\
\hline \multirow[t]{5}{*}{196} & $\sigma(q \bar{q})[\mathrm{pb}]$ & $20.730 \pm 0.330$ & 20.127 \\
\hline & $\sigma\left(\mu^{+} \mu^{-}\right)[\mathrm{pb}]$ & $2.965 \pm 0.106$ & 2.962 \\
\hline & $\sigma\left(\tau^{+} \tau^{-}\right)[\mathrm{pb}]$ & $3.015 \pm 0.139$ & 2.962 \\
\hline & $\mathrm{A}_{\mathrm{fb}}\left(\mu^{+} \mu^{-}\right)$ & $0.579 \pm 0.031$ & 0.562 \\
\hline & $\mathrm{A}_{\mathrm{fb}}\left(\tau^{+} \tau^{-}\right)$ & $0.489 \pm 0.045$ & 0.562 \\
\hline \multirow[t]{5}{*}{200} & $\sigma(q \bar{q})[\mathrm{pb}]$ & $19.376 \pm 0.306$ & 19.085 \\
\hline & $\sigma\left(\mu^{+} \mu^{-}\right)[\mathrm{pb}]$ & $3.038 \pm 0.104$ & 2.834 \\
\hline & $\sigma\left(\tau^{+} \tau^{-}\right)[\mathrm{pb}]$ & $2.995 \pm 0.135$ & 2.833 \\
\hline & $\mathrm{A}_{\mathrm{fb}}\left(\mu^{+} \mu^{-}\right)$ & $0.518 \pm 0.031$ & 0.558 \\
\hline & $\mathrm{A}_{\mathrm{fb}}\left(\tau^{+} \tau^{-}\right)$ & $0.546 \pm 0.043$ & 0.558 \\
\hline \multirow[t]{5}{*}{202} & $\sigma(q \bar{q})[\mathrm{pb}]$ & $19.291 \pm 0.425$ & 18.572 \\
\hline & $\sigma\left(\mu^{+} \mu^{-}\right)[\mathrm{pb}]$ & $2.621 \pm 0.139$ & 2.770 \\
\hline & $\sigma\left(\tau^{+} \tau^{-}\right)[\mathrm{pb}]$ & $2.806 \pm 0.183$ & 2.769 \\
\hline & $\mathrm{A}_{\mathrm{fb}}\left(\mu^{+} \mu^{-}\right)$ & $0.543 \pm 0.048$ & 0.556 \\
\hline & $\mathrm{A}_{\mathrm{fb}}\left(\tau^{+} \tau^{-}\right)$ & $0.580 \pm 0.060$ & 0.556 \\
\hline
\end{tabular}


TABLE XII: Combined LEP 2 Data on the $R_{b}$ and $R_{c}$ along with the Standard Model predictions.

\begin{tabular}{|c|c|c|}
\hline$\sqrt{s}(\mathrm{GeV})$ & $R_{b}$ & $R_{c}$ \\
\hline \multirow[t]{2}{*}{133} & $0.1809 \pm 0.0133$ & - \\
\hline & $(0.1853)$ & - \\
\hline \multirow[t]{2}{*}{167} & $0.1479 \pm 0.0127$ & - \\
\hline & $(0.1708)$ & - \\
\hline \multirow[t]{2}{*}{183} & $0.1616 \pm 0.0101$ & $0.270 \pm 0.043$ \\
\hline & $(0.1671)$ & $(0.250)$ \\
\hline \multirow[t]{2}{*}{189} & $0.1559 \pm 0.0066$ & $0.241 \pm 0.024$ \\
\hline & $(0.1660)$ & $(0.252)$ \\
\hline \multirow[t]{2}{*}{192} & $0.1688 \pm 0.0187$ & - \\
\hline & $(0.1655)$ & - \\
\hline \multirow[t]{2}{*}{196} & $0.1577 \pm 0.0109$ & - \\
\hline & $(0.1648)$ & - \\
\hline \multirow[t]{2}{*}{200} & $0.1621 \pm 0.0111$ & - \\
\hline & $(0.1642)$ & - \\
\hline \multirow[t]{2}{*}{202} & $0.1873 \pm 0.0177$ & - \\
\hline & $(0.1638)$ & - \\
\hline \multirow[t]{2}{*}{206} & $0.1696 \pm 0.0182$ & - \\
\hline & $(0.1633)$ & - \\
\hline
\end{tabular}


TABLE XIII: The LEP $2 e^{+} e^{-} \rightarrow e^{+} e^{-}$production cross sections.

\begin{tabular}{|c|c|c|}
\hline$\sqrt{s}(\mathrm{GeV})$ & $\sigma_{e e}(\mathrm{pb})$ & $\sigma_{e e}^{\mathrm{SM}}(\mathrm{pb})$ \\
\hline \multicolumn{3}{|c|}{ ALEPH } \\
\hline 130 & $191.3 \pm 6.2 \pm 3.5$ & 186.7 \\
\hline 136 & $162.2 \pm 5.6 \pm 3.5$ & 167.3 \\
\hline 161 & $119.7 \pm 3.7 \pm 2.3$ & 119.0 \\
\hline 172 & $107.8 \pm 3.5 \pm 2.1$ & 102.5 \\
\hline 183 & $90.9 \pm 1.4 \pm 1.7$ & 90.9 \\
\hline 189 & $84 \pm 0.72 \pm 0.71$ & 87.25 \\
\hline 192 & $80.00 \pm 1.90 \pm 0.87$ & 81.9 \\
\hline 196 & $78.74 \pm 1.10 \pm 0.76$ & 79.2 \\
\hline 200 & $73.95 \pm 1.02 \pm 0.70$ & 76.3 \\
\hline 202 & $75.42 \pm 1.50 \pm 0.80$ & 74.6 \\
\hline 204.9 & $75.33 \pm 1.20 \pm 0.90$ & 74.36 \\
\hline 206.7 & $73.40 \pm 0.91 \pm 0.70$ & 74.50 \\
\hline \multicolumn{3}{|c|}{ DELPHI } \\
\hline 130.2 & $42.0 \pm 4.0 \pm 0.80$ & 48.7 \\
\hline 136.2 & $47.1 \pm 4.2 \pm 0.71$ & 44.6 \\
\hline 161.3 & $27.1 \pm 1.8 \pm 0.41$ & 31.9 \\
\hline 172.1 & $30.3 \pm 1.9 \pm 0.36$ & 28.0 \\
\hline 183 & $25.6 \pm 0.8$ & 24.7 \\
\hline 189 & $22.6 \pm 0.4$ & 23.1 \\
\hline 192 & $24.03 \pm 1.03$ & 22.29 \\
\hline 196 & $22.43 \pm 0.56$ & 21.36 \\
\hline 200 & $20.56 \pm 0.51$ & 20.52 \\
\hline 202 & $21.30 \pm 0.75$ & 20.08 \\
\hline \multicolumn{3}{|c|}{ L3 } \\
\hline 130.1 & $45.0 \pm 2.7 \pm 0.2$ & 49.7 \\
\hline 136.1 & $43.6 \pm 2.8 \pm 0.2$ & 45.4 \\
\hline 161.3 & $31.1 \pm 1.8 \pm 0.9$ & 32.4 \\
\hline 172.3 & $26.7 \pm 1.8 \pm 0.8$ & 28.3 \\
\hline 182.7 & $25.6 \pm 0.7 \pm 0.1$ & 25.0 \\
\hline 188.7 & $23.5 \pm 0.4 \pm 0.1$ & 23.4 \\
\hline \multicolumn{3}{|c|}{ OPAL } \\
\hline 130.25 & $615 \pm 16 \pm 8$ & 645 \\
\hline 136.22 & $580 \pm 15 \pm 8$ & 592 \\
\hline 161.34 & $434 \pm 7 \pm 5$ & 425 \\
\hline 172.12 & $365 \pm 6 \pm 5$ & 375 \\
\hline 183 & $333 \pm 3 \pm 4$ & 333 \\
\hline 189 & $304.6 \pm 1.3 \pm 1.4$ & 311.6 \\
\hline 192 & $301.4 \pm 3.3 \pm 1.5$ & 299.4 \\
\hline 196 & $285.8 \pm 2.0 \pm 1.5$ & 287.7 \\
\hline 200 & $273.0 \pm 1.9 \pm 1.4$ & 276.3 \\
\hline 202 & $272.0 \pm 2.8 \pm 1.4$ & 270.6 \\
\hline
\end{tabular}


TABLE XIV: The $e^{+} e^{-} \rightarrow e^{+} e^{-}$differential cross section $d \sigma / d \cos \theta^{*}$ measured by ALEPH.

\begin{tabular}{|c|c|c|c|c|c|c|}
\hline \multirow[t]{2}{*}{$\cos \theta^{*}$ range } & \multicolumn{2}{|c|}{$130 \mathrm{GeV}$} & \multicolumn{2}{|c|}{$136 \mathrm{GeV}$} & \multicolumn{2}{|c|}{$161 \mathrm{GeV}$} \\
\hline & $\sigma(\mathrm{pb})$ & $\sigma_{\mathrm{SM}}(\mathrm{pb})$ & $\sigma(\mathrm{pb})$ & $\sigma_{\mathrm{SM}}(\mathrm{pb})$ & $\sigma(\mathrm{pb})$ & $\sigma_{\mathrm{SM}}(\mathrm{pb})$ \\
\hline$-0.9--0.7$ & $0.19 \pm 0.34$ & 0.37 & $0.73 \pm 0.20$ & 0.22 & $0.46 \pm 0.21$ & 0.37 \\
\hline$-0.7--0.5$ & $1.41 \pm 0.35$ & 0.55 & $1.16 \pm 0.36$ & 0.62 & $0.88 \pm 0.21$ & 0.44 \\
\hline$-0.5--0.3$ & $1.36 \pm 0.45$ & 1.09 & $0.54 \pm 0.35$ & 0.49 & $0.55 \pm 0.28$ & 0.79 \\
\hline$-0.3--0.1$ & $1.23 \pm 0.48$ & 1.19 & $0.52 \pm 0.41$ & 0.89 & $0.39 \pm 0.26$ & 0.62 \\
\hline$-0.1-0.1$ & $2.60 \pm 0.69$ & 2.45 & $1.46 \pm 0.62$ & 2.09 & $1.24 \pm 0.40$ & 1.43 \\
\hline $0.1-0.3$ & $3.78 \pm 0.83$ & 3.82 & $2.09 \pm 0.74$ & 2.96 & $2.37 \pm 0.47$ & 2.07 \\
\hline $0.3-0.5$ & $8.88 \pm 1.18$ & 7.36 & $6.68 \pm 1.08$ & 6.13 & $5.35 \pm 0.73$ & 4.95 \\
\hline $0.5-0.7$ & $21.63 \pm 2.12$ & 22.20 & $16.58 \pm 1.97$ & 20.50 & $14.38 \pm 1.27$ & 14.10 \\
\hline \multirow[t]{2}{*}{$0.7-0.9$} & $149.61 \pm 6.22$ & 148.0 & $132.55 \pm 5.85$ & 133.0 & $93.76 \pm 3.76$ & 94.20 \\
\hline & \multicolumn{2}{|c|}{$172 \mathrm{GeV}$} & \multicolumn{2}{|c|}{$183 \mathrm{GeV}$} & & \\
\hline$-0.9--0.7$ & $0.32 \pm 0.19$ & 0.28 & $0.24 \pm 0.07$ & 0.21 & & \\
\hline$-0.7--0.5$ & $0.88 \pm 0.19$ & 0.34 & $0.29 \pm 0.07$ & 0.25 & & \\
\hline$-0.5--0.3$ & $0.66 \pm 0.24$ & 0.58 & $0.46 \pm 0.10$ & 0.51 & & \\
\hline$-0.3--0.1$ & $0.61 \pm 0.23$ & 0.44 & $0.71 \pm 0.12$ & 0.64 & & \\
\hline$-0.1-0.1$ & $0.95 \pm 0.36$ & 1.23 & $0.83 \pm 0.14$ & 0.90 & & \\
\hline $0.1-0.3$ & $1.80 \pm 0.47$ & 1.93 & $1.42 \pm 0.20$ & 1.83 & & \\
\hline $0.3-0.5$ & $4.92 \pm 0.71$ & 4.24 & $3.90 \pm 0.29$ & 3.66 & & \\
\hline $0.5-0.7$ & $13.07 \pm 1.20$ & 12.40 & $12.47 \pm 0.56$ & 11.10 & & \\
\hline $0.7-0.9$ & $84.61 \pm 3.51$ & 81.10 & $71.90 \pm 1.86$ & 71.80 & & \\
\hline
\end{tabular}


TABLE XV: The $e^{+} e^{-} \rightarrow \mu^{+} \mu^{-}$and $e^{+} e^{-} \rightarrow \tau^{+} \tau^{-}$differential cross sections $d \sigma / d \cos \theta^{*}$ measured by ALEPH.

\begin{tabular}{|c|c|c|c|c|c|c|c|}
\hline \multirow[t]{2}{*}{$\cos \theta^{*}$ range } & \multicolumn{7}{|c|}{$d \sigma / d \cos \theta^{*}(\mathrm{pb})$} \\
\hline & $189 \mathrm{GeV}$ & $192 \mathrm{GeV}$ & $196 \mathrm{GeV}$ & $200 \mathrm{GeV}$ & $202 \mathrm{GeV}$ & $204.9 \mathrm{GeV}$ & $206.7 \mathrm{GeV}$ \\
\hline \multicolumn{8}{|c|}{$\mu^{+} \mu^{-}$} \\
\hline$-0.95--0.8$ & $0.69 \pm 0.17$ & $0.23 \pm 0.25$ & $0.61 \pm 0.24$ & $0.64 \pm 0.23$ & $0.83 \pm 0.38$ & $0.42 \pm 0.20$ & $0.51 \pm 0.18$ \\
\hline$-0.8--0.6$ & $0.31 \pm 0.10$ & $0.17 \pm 0.18$ & $0.56 \pm 0.20$ & $0.58 \pm 0.19$ & $0.10 \pm 0.13$ & $0.29 \pm 0.15$ & $0.43 \pm 0.14$ \\
\hline$-0.6--0.4$ & $0.38 \pm 0.11$ & $1.07 \pm 0.45$ & $0.51 \pm 0.19$ & $0.90 \pm 0.24$ & $0.86 \pm 0.33$ & $0.17 \pm 0.13$ & $0.25 \pm 0.11$ \\
\hline$-0.4--0.2$ & $0.75 \pm 0.16$ & $0.72 \pm 0.37$ & $0.58 \pm 0.20$ & $1.02 \pm 0.26$ & $0.48 \pm 0.26$ & $0.56 \pm 0.31$ & $1.00 \pm 0.21$ \\
\hline$-0.2-0.0$ & $1.11 \pm 0.19$ & $0.89 \pm 0.42$ & $0.88 \pm 0.25$ & $1.20 \pm 0.28$ & $0.73 \pm 0.32$ & $0.94 \pm 0.25$ & $0.60 \pm 0.16$ \\
\hline $0.0-0.2$ & $1.07 \pm 0.19$ & $1.80 \pm 0.59$ & $1.09 \pm 0.28$ & $1.59 \pm 0.32$ & $1.22 \pm 0.41$ & $1.00 \pm 0.27$ & $1.06 \pm 0.21$ \\
\hline $0.2-0.4$ & $2.09 \pm 0.26$ & $0.88 \pm 0.42$ & $1.89 \pm 0.37$ & $1.97 \pm 0.36$ & $1.70 \pm 0.48$ & $1.17 \pm 0.29$ & $1.61 \pm 0.26$ \\
\hline $0.4-0.6$ & $1.93 \pm 0.25$ & $2.28 \pm 0.66$ & $2.38 \pm 0.40$ & $1.78 \pm 0.34$ & $2.42 \pm 0.56$ & $1.64 \pm 0.34$ & $1.94 \pm 0.28$ \\
\hline $0.6-0.8$ & $3.24 \pm 0.32$ & $3.96 \pm 0.85$ & $2.84 \pm 0.44$ & $3.03 \pm 0.43$ & $2.22 \pm 0.54$ & $1.65 \pm 0.35$ & $2.84 \pm 0.35$ \\
\hline $0.8-0.95$ & $3.80 \pm 0.40$ & $3.83 \pm 0.99$ & $3.12 \pm 0.54$ & $3.35 \pm 0.54$ & $3.81 \pm 0.82$ & $2.33 \pm 0.48$ & $2.95 \pm 0.42$ \\
\hline \multicolumn{8}{|c|}{$\tau^{+} \tau^{-}$} \\
\hline$-0.95--0.8$ & $0.43 \pm 0.33$ & $-0.35 \pm 0.42$ & $0.80 \pm 0.49$ & $0.76 \pm 0.47$ & $0.94 \pm 0.77$ & $0.65 \pm 0.46$ & $0.35 \pm 0.27$ \\
\hline$-0.8--0.6$ & $0.49 \pm 0.18$ & $0.36 \pm 0.47$ & $1.02 \pm 0.39$ & $0.93 \pm 0.35$ & $0.09 \pm 0.28$ & $0.50 \pm 0.28$ & $0.38 \pm 0.21$ \\
\hline$-0.6--0.4$ & $0.17 \pm 0.13$ & $0.18 \pm 0.33$ & $-0.03 \pm 0.17$ & $0.70 \pm 0.32$ & $0.36 \pm 0.34$ & $0.39 \pm 0.27$ & $0.38 \pm 0.21$ \\
\hline$-0.4--0.2$ & $0.99 \pm 0.24$ & $1.64 \pm 0.75$ & $0.58 \pm 0.29$ & $0.68 \pm 0.31$ & $0.43 \pm 0.44$ & $0.21 \pm 0.23$ & $0.60 \pm 0.25$ \\
\hline$-0.2-0.0$ & $0.82 \pm 0.24$ & $0.76 \pm 0.58$ & $0.82 \pm 0.36$ & $1.27 \pm 0.42$ & $0.45 \pm 0.42$ & $0.20 \pm 0.25$ & $0.37 \pm 0.20$ \\
\hline $0.0-0.2$ & $0.70 \pm 0.24$ & $1.98 \pm 0.83$ & $0.72 \pm 0.34$ & $1.44 \pm 0.43$ & $1.20 \pm 0.59$ & $1.14 \pm 0.41$ & $1.37 \pm 0.34$ \\
\hline $0.2-0.4$ & $1.98 \pm 0.34$ & $1.90 \pm 0.85$ & $2.50 \pm 0.58$ & $1.71 \pm 0.48$ & $2.01 \pm 0.72$ & $1.73 \pm 0.50$ & $1.27 \pm 0.34$ \\
\hline $0.4-0.6$ & $2.16 \pm 0.37$ & $2.65 \pm 0.98$ & $1.68 \pm 0.48$ & $1.84 \pm 0.49$ & $0.94 \pm 0.58$ & $1.43 \pm 0.48$ & $1.35 \pm 0.35$ \\
\hline $0.6-0.8$ & $2.40 \pm 0.39$ & $2.55 \pm 0.99$ & $2.65 \pm 0.65$ & $2.86 \pm 0.63$ & $4.71 \pm 1.13$ & $1.60 \pm 0.52$ & $1.85 \pm 0.41$ \\
\hline $0.8-0.95$ & $7.09 \pm 1.22$ & $3.74 \pm 1.89$ & $6.24 \pm 1.24$ & $2.65 \pm 1.36$ & $3.33 \pm 2.09$ & $3.09 \pm 1.08$ & $2.67 \pm 0.72$ \\
\hline
\end{tabular}


TABLE XVI: Differential cross section $d \sigma / d \cos \theta^{*}$ for the $e^{+} e^{-} \rightarrow e^{+} e^{-}, \mu^{+} \mu^{-}, \tau^{+} \tau^{-}, q \bar{q}$ production measured by OPAL.

\begin{tabular}{|c|c|c|c|c|c|c|c|c|}
\hline \multirow[t]{2}{*}{$\cos \theta^{*}$ range } & \multicolumn{8}{|c|}{$d \sigma / d \cos \theta^{*}(\mathrm{pb})$} \\
\hline & $130.12 \mathrm{GeV}$ & $136.08 \mathrm{GeV}$ & $182.69 \mathrm{GeV}$ & $189 \mathrm{GeV}$ & $192 \mathrm{GeV}$ & $196 \mathrm{GeV}$ & $200 \mathrm{GeV}$ & $202 \mathrm{GeV}$ \\
\hline & \multicolumn{8}{|c|}{$e^{+} e^{-}$} \\
\hline$-0.9--0.7$ & $\begin{array}{l}4_{-2}^{+3} \\
-2\end{array}$ & $5_{-2}^{+3}$ & $1.2_{-0.3}^{+0.4}$ & $1.4 \pm 0.2$ & $1.7_{-0.5}^{+0.7}$ & $1.4 \pm 0.3$ & $1.6 \pm 0.3$ & $2.3_{-0.6}^{+0.7}$ \\
\hline$-0.7--0.5$ & $4 \begin{array}{l}+3 \\
-2\end{array}$ & $4 \stackrel{+3}{-2}$ & $2.1 \pm 0.4$ & $2.0 \pm 0.2$ & $1.9_{-0.6}^{+0.8}$ & $1.6 \pm 0.3$ & $1.9 \pm 0.3$ & $1.6_{-0.5}^{+0.6}$ \\
\hline$-0.5--0.3$ & $6{ }_{-2}^{+3}$ & $8 \stackrel{+4}{-3}$ & $2.3 \pm 0.5$ & $2.4 \pm 0.3$ & $1.9_{-0.6}^{+0.8}$ & $2.6 \pm 0.4$ & $2.3 \pm 0.4$ & $3.2 \pm 0.7$ \\
\hline$-0.3--0.1$ & $6{ }_{-2}^{+4}$ & $9 \stackrel{+4}{-3}$ & $4.8 \pm 0.7$ & $3.0 \pm 0.3$ & $1.5_{-0.5}^{+0.7}$ & $2.7 \pm 0.4$ & $2.4 \pm 0.4$ & $2.5_{-0.6}^{+0.7}$ \\
\hline$-0.1-0.1$ & $13_{-4}^{+5}$ & $8 \stackrel{+4}{-3}$ & $6.1 \pm 0.7$ & $4.3 \pm 0.3$ & $5.4 \pm 1.0$ & $3.7 \pm 0.5$ & $5.2 \pm 0.6$ & $4.4 \pm 0.8$ \\
\hline $0.1-0.3$ & $23 \pm 5$ & $18 \pm 4$ & $9.5 \pm 0.9$ & $8.3 \pm 0.5$ & $9.6 \pm 1.3$ & $8.3 \pm 0.7$ & $7.4 \pm 0.7$ & $8.5 \pm 1.1$ \\
\hline $0.3-0.5$ & $45 \pm 7$ & $35 \pm 6$ & $21.1 \pm 1.4$ & $19.3 \pm 0.7$ & $19.3 \pm 1.8$ & $17.0 \pm 1.1$ & $17.0 \pm 1.1$ & $14.5 \pm 1.4$ \\
\hline $0.5-0.7$ & $113 \pm 11$ & $122 \pm 10$ & $62 \pm 2$ & $61.4 \pm 1.4$ & $59.0 \pm 3.2$ & $56.7 \pm 2.0$ & $55.6 \pm 2.0$ & $54.6 \pm 2.8$ \\
\hline \multirow[t]{2}{*}{$0.7-0.9$} & $839 \pm 30$ & $725 \pm 27$ & $458 \pm 8$ & $415 \pm 5$ & $396 \pm 9$ & $388 \pm 6$ & $369 \pm 6$ & $361 \pm 8$ \\
\hline & \multicolumn{8}{|c|}{$\mu^{+} \mu^{-}$} \\
\hline$-1.0--0.8$ & $0_{-1}^{+3}$ & $-1{ }_{-0}^{+1}$ & $0.4_{-0.2}^{+0.4}$ & $0.67_{-0.17}^{+0.21}$ & $1.2_{-0.5}^{+0.8}$ & $0.19_{-0.12}^{+0.24}$ & $0.52_{-0.22}^{+0.33}$ & $1.1_{-0.5}^{+0.7}$ \\
\hline$-0.8--0.6$ & $\begin{array}{l}+3 \\
-2\end{array}$ & $2+3$ & $0.7_{-0.3}^{+0.4}$ & $0.36_{-0.11}^{+0.14}$ & $0.7_{-0.4}^{+0.6}$ & $0.35_{-0.17}^{+0.26}$ & $0.20_{-0.13}^{+0.22}$ & $0.2_{-0.1}^{+0.4}$ \\
\hline$-0.6--0.4$ & $0 \stackrel{+2}{-1}$ & $0_{-1}^{+2}$ & $0.5_{-0.2}^{+0.4}$ & $0.50 \stackrel{+0.16}{-0.13}$ & $0.3_{-0.3}^{+0.5}$ & $0.84+0.34$ & $0.49_{-0.20}^{+0.29}$ & $0.2_{-0.2}^{+0.4}$ \\
\hline$-0.4--0.2$ & $6 \stackrel{-3}{+4}_{-3}$ & $5 \stackrel{+3}{-2}$ & $0.9 \stackrel{+0.4}{+0.3}$ & $0.66 \pm 0.14$ & $0.8_{-0.4}^{+0.6}$ & $0.60_{-0.20}^{+0.28}$ & $0.56_{-0.20}^{+0.28}$ & $0.3_{-0.2}^{+0.4}$ \\
\hline$-0.2-0.0$ & $3 \begin{array}{l}+3 \\
-2\end{array}$ & $1 \stackrel{+2}{-1}$ & $1.2_{-0.3}^{+0.5}$ & $1.33 \pm 0.20$ & $2.0 \begin{array}{l}+0.8 \\
-0.6\end{array}$ & $0.55_{-0.20}^{+0.28}$ & $0.83_{-0.24}^{+0.32}$ & $1.2_{-0.4}^{+0.6}$ \\
\hline $0.0-0.2$ & $2+2$ & $3+2$ & $1.8 \pm 0.4$ & $1.20 \pm 0.19$ & $1.0_{-0.4}^{+0.6}$ & $1.5 \pm 0.3$ & $1.2_{-0.3}^{+0.4}$ & $1.0_{-0.4}^{+0.6}$ \\
\hline $0.2-0.4$ & $6 \stackrel{+4}{-3}$ & $6{ }_{-2}^{+3}$ & $2.4 \pm 0.5$ & $1.85 \pm 0.24$ & $2.1+0.8$ & $1.6 \pm 0.3$ & $2.0 \pm 0.4$ & $1.0_{-0.4}^{+0.6}$ \\
\hline $0.4-0.6$ & $5_{-2}^{+4}$ & $10 \stackrel{+4}{-4}$ & $1.9 \pm 0.5$ & $2.04 \pm 0.27$ & $1.5_{-0.6}^{+0.8}$ & $2.0 \pm 0.4$ & $1.4 \pm 0.3$ & $1.2_{-0.5}^{+0.6}$ \\
\hline $0.6-0.8$ & $5_{-3}^{+4}$ & $9_{-3}^{+4}$ & $2.5 \pm 0.5$ & $2.64 \pm 0.30$ & $2.5 \begin{array}{l}+1.0 \\
-0.8\end{array}$ & $3.0 \pm 0.5$ & $2.8 \pm 0.5$ & $2.5_{-0.7}^{+0.8}$ \\
\hline \multirow[t]{2}{*}{$0.8-1.0$} & $9 \stackrel{+5}{-4}$ & $18{ }_{-5}^{+7}$ & $4.7 \pm 0.9$ & $3.96 \pm 0.43$ & $1.9_{-0.8}^{+1.1}$ & $3.9 \pm 0.6$ & $3.7 \pm 0.6$ & $2.8+1.0$ \\
\hline & \multicolumn{8}{|c|}{$\tau^{+} \tau^{-}$} \\
\hline$-1.0--0.8$ & $\begin{array}{c}+10 \\
-1+0\end{array}$ & $\begin{array}{l}+9 \\
-1+0\end{array}$ & $0.7_{-0.5}^{+0.9}$ & $0.99_{-0.33}^{+0.44}$ & $0.9_{-0.9}^{+1.7}$ & $1.2_{-0.5}^{+0.8}$ & $0.6_{-0.4}^{+0.7}$ & $2.4_{-1.1}^{+1.7}$ \\
\hline$-0.8--0.6$ & $0 \begin{array}{l}+1 \\
-0\end{array}$ & $0 \begin{array}{l}+1 \\
-0\end{array}$ & $0.0_{-0.1}^{+0.3}$ & $0.39_{-0.15}^{+0.19}$ & $0.1+0.6$ & $1.3_{-0.4}^{+0.5}$ & $0.2 \begin{array}{l}+0.3 \\
-0.2\end{array}$ & $0.4_{-0.3}^{+0.6}$ \\
\hline$-0.6--0.4$ & $-1 \stackrel{+2}{-0}$ & $2+3$ & $0.0_{-0.1}^{+0.3}$ & $0.75 \pm 0.18$ & $0.7_{-0.4}^{+0.7}$ & $0.6_{-0.2}^{+0.4}$ & $0.5_{-0.2}^{+0.3}$ & $0.7_{-0.4}^{+0.7}$ \\
\hline$-0.4--0.2$ & $2 \stackrel{+4}{-2}$ & 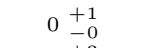 & $0.7_{-0.3}^{+0.5}$ & $0.76 \pm 0.18$ & $0.3_{-0.3}^{+0.7}$ & $0.7_{-0.3}^{+0.4}$ & $1.0_{-0.3}^{+0.4}$ & $0.9_{-0.4}^{+0.7}$ \\
\hline$-0.2-0.0$ & $3_{-2}^{+4}$ & 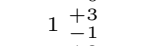 & $1.5_{-0.5}^{+0.6}$ & $0.84 \pm 0.19$ & $0.4_{-0.3}^{+0.6}$ & $0.8_{-0.3}^{+0.4}$ & $1.0_{-0.3}^{+0.4}$ & $0.5_{-0.3}^{+0.6}$ \\
\hline $0.0-0.2$ & $2+2$ & $4{ }_{-2}^{+3}$ & $1.6_{-0.5}^{+0.6}$ & $1.68 \pm 0.27$ & $2.0_{-0.7}^{+1.0}$ & $1.1_{-0.3}^{+0.4}$ & $1.4_{-0.3}^{+0.4}$ & $0.9_{-0.4}^{+0.7}$ \\
\hline $0.2-0.4$ & $1 \stackrel{+4}{-2}$ & $4+2$ & $1.5_{-0.5}^{+0.6}$ & $2.00 \pm 0.30$ & $2.0+0.7$ & $1.3 \stackrel{+0.5}{-0.4}$ & $2.0 \pm 0.5$ & $1.5_{-0.6}^{+0.8}$ \\
\hline $0.4-0.6$ & $7_{-3}^{+5}$ & $7_{-3}^{+4}$ & $2.5 \pm 0.6$ & $2.52 \pm 0.33$ & $1.9_{-0.7}^{+1.0}$ & $2.2 \pm 0.5$ & $2.4 \pm 0.5$ & $3.7_{-0.9}^{+1.1}$ \\
\hline $0.6-0.8$ & $7_{-3}^{+5}$ & $8 \stackrel{+5}{-3}$ & $4.3 \pm 0.8$ & $3.29 \pm 0.40$ & $3.9+1.3$ & $2.4 \pm 0.5$ & $3.7 \pm 0.6$ & $3.4_{-0.9}^{+1.1}$ \\
\hline \multirow[t]{2}{*}{$0.8-1.0$} & $14_{-11}^{+19}$ & $7 \stackrel{+15}{+7}$ & $3.9 \stackrel{+1.6}{+1.3}$ & $5.1 \pm 0.8$ & $6.3_{-2.0}^{+2.7}$ & $4.8 \pm 1.1$ & $5.5 \pm 1.2$ & $3.2_{-1.3}^{+1.9}$ \\
\hline & \multicolumn{8}{|c|}{$q \bar{q}$} \\
\hline $0.0-0.1$ & $70 \pm 12$ & $48 \pm 9$ & $17.0 \pm 1.8$ & $17.5 \pm 1.0$ & & & & \\
\hline $0.1-0.2$ & $52 \pm 9$ & $64 \pm 10$ & $17.6 \pm 1.8$ & $17.7 \pm 1.1$ & & & & \\
\hline $0.2-0.3$ & $70 \pm 11$ & $56 \pm 10$ & $17.7 \pm 1.9$ & $16.8 \pm 1.0$ & & & & \\
\hline $0.3-0.4$ & $70 \pm 11$ & $63 \pm 10$ & $19.9 \pm 2.0$ & $18.2 \pm 1.1$ & & & & \\
\hline $0.4-0.5$ & $64 \pm 10$ & $44 \pm 9$ & $23.1 \pm 2.1$ & $18.8 \pm 1.1$ & & & & \\
\hline $0.5-0.6$ & $79 \pm 12$ & $39 \pm 8$ & $24.6 \pm 2.2$ & $21.6 \pm 1.2$ & & & & \\
\hline $0.6-0.7$ & $81 \pm 12$ & $78 \pm 11$ & $27.2 \pm 2.3$ & $24.2 \pm 1.2 \mathrm{y}$ & & & & \\
\hline $0.7-0.8$ & $94 \pm 13$ & $81 \pm 11$ & $26.1 \pm 2.2$ & $26.0 \pm 1.3$ & & & & \\
\hline $0.8-0.9$ & $85 \pm 12$ & $82 \pm 11$ & $31.2 \pm 2.4$ & $27.7 \pm 1.3$ & & & & \\
\hline $0.9-1.0$ & $160 \pm 23$ & $123 \pm 19$ & $32.0 \pm 3.2$ & $31.4 \pm 1.7$ & & & & \\
\hline
\end{tabular}


TABLE XVII: Differential cross section $d \sigma / d \cos \theta^{*}$ for the $e^{+} e^{-} \rightarrow \mu^{+} \mu^{-}, \tau^{+} \tau^{-}$production measured by DELPHI. For $\sqrt{s}=183 \mathrm{GeV}$ the $\cos \theta^{*}$ range for $\mu^{+} \mu^{-}$channel is from -0.94 to 0.94 .

\begin{tabular}{|c|c|c|c|c|c|c|}
\hline \multirow[t]{2}{*}{$\cos \theta^{*}$ range } & \multicolumn{6}{|c|}{$d \sigma / d \cos \theta^{*}(\mathrm{pb})$} \\
\hline & $183 \mathrm{GeV}$ & $189 \mathrm{GeV}$ & $192 \mathrm{GeV}$ & $196 \mathrm{GeV}$ & $200 \mathrm{GeV}$ & $202 \mathrm{GeV}$ \\
\hline & \multicolumn{6}{|c|}{$\mu^{+} \mu^{-}$} \\
\hline$-0.97--0.8$ & $.000 \pm .178 \pm .013$ & $.495 \pm .143 \pm .008$ & $.000 \pm .331 \pm .005$ & $.716 \pm .253 \pm .018$ & $.560 \pm .211 \pm .016$ & $1.077 \pm .407 \pm .029$ \\
\hline$-0.8--0.6$ & $.514 \pm .230 \pm .013$ & $.478 \pm .128 \pm .008$ & $.202 \pm .202 \pm .005$ & $.520 \pm .196 \pm .013$ & $.266 \pm .133 \pm .007$ & $.585 \pm .292 \pm .017$ \\
\hline$-0.6--0.4$ & $.989 \pm .313 \pm .024$ & $.448 \pm .120 \pm .007$ & $.814 \pm .407 \pm .020$ & $.614 \pm .205 \pm .015$ & $.885 \pm .236 \pm .023$ & $.259 \pm .183 \pm .007$ \\
\hline$-0.4--0.2$ & $.972 \pm .307 \pm .023$ & $.391 \pm .113 \pm .006$ & $.385 \pm .272 \pm .009$ & $.208 \pm .120 \pm .005$ & $.699 \pm .211 \pm .018$ & $.249 \pm .176 \pm .007$ \\
\hline$-0.2-0.0$ & $1.298 \pm .360 \pm .032$ & $1.287 \pm .212 \pm .021$ & $1.068 \pm .477 \pm .027$ & $.875 \pm .253 \pm .022$ & $1.053 \pm .263 \pm .029$ & $.392 \pm .226 \pm .011$ \\
\hline $0.0-0.2$ & $1.591 \pm .398 \pm .039$ & $1.129 \pm .197 \pm .018$ & $.619 \pm .357 \pm .016$ & $1.459 \pm .318 \pm .035$ & $1.301 \pm .291 \pm .035$ & $.515 \pm .257 \pm .014$ \\
\hline $0.2-0.4$ & $1.605 \pm .401 \pm .039$ & $1.908 \pm .248 \pm .029$ & $2.595 \pm .720 \pm .063$ & $1.279 \pm .301 \pm .031$ & $1.694 \pm .326 \pm .044$ & $2.385 \pm .562 \pm .065$ \\
\hline $0.4-0.6$ & $3.377 \pm .579 \pm .081$ & $2.445 \pm .290 \pm .039$ & $1.859 \pm .620 \pm .046$ & $2.171 \pm .396 \pm .054$ & $2.957 \pm .436 \pm .079$ & $1.325 \pm .419 \pm .036$ \\
\hline $0.6-0.8$ & $2.466 \pm .503 \pm .061$ & $2.927 \pm .325 \pm .048$ & $2.592 \pm .748 \pm .067$ & $3.337 \pm .503 \pm .085$ & $2.877 \pm .439 \pm .079$ & $2.051 \pm .530 \pm .057$ \\
\hline \multirow[t]{2}{*}{$0.8-0.97$} & $4.978 \pm .841 \pm .119$ & $3.986 \pm .413 \pm .065$ & $3.191 \pm .885 \pm .080$ & $2.791 \pm .493 \pm .070$ & $3.656 \pm .533 \pm .099$ & $3.125 \pm .717 \pm .088$ \\
\hline & \multicolumn{6}{|c|}{$\tau^{+} \tau^{-}$} \\
\hline$-0.96--0.8$ & $-0.13 \pm 0.24 \pm 0.04$ & $0.58 \pm 0.34 \pm 0.05$ & $0.70 \pm 0.77 \pm 0.06$ & $0.70 \pm 0.44 \pm 0.06$ & $0.10 \pm 0.42 \pm 0.06$ & $-0.15 \pm 0.60 \pm 0.06$ \\
\hline$-0.8--0.6$ & $0.48 \pm 0.31 \pm 0.04$ & $0.12 \pm 0.13 \pm 0.03$ & $0.31 \pm 0.48 \pm 0.05$ & $0.18 \pm 0.27 \pm 0.05$ & $1.01 \pm 0.26 \pm 0.05$ & $0.89 \pm 0.38 \pm 0.05$ \\
\hline$-0.6--0.4$ & $0.52 \pm 0.28 \pm 0.04$ & $0.48 \pm 0.16 \pm 0.04$ & $0.49 \pm 0.43 \pm 0.06$ & $0.97 \pm 0.25 \pm 0.06$ & $0.53 \pm 0.24 \pm 0.06$ & $0.45 \pm 0.34 \pm 0.06$ \\
\hline$-0.4--0.2$ & $0.56 \pm 0.30 \pm 0.04$ & $0.67 \pm 0.19 \pm 0.05$ & $0.50 \pm 0.50 \pm 0.07$ & $0.69 \pm 0.29 \pm 0.07$ & $0.53 \pm 0.27 \pm 0.07$ & $0.45 \pm 0.39 \pm 0.07$ \\
\hline$-0.2-0.0$ & $1.62 \pm 0.54 \pm 0.12$ & $0.75 \pm 0.22 \pm 0.06$ & $0.94 \pm 0.60 \pm 0.09$ & $1.28 \pm 0.34 \pm 0.09$ & $1.06 \pm 0.33 \pm 0.09$ & $1.40 \pm 0.48 \pm 0.09$ \\
\hline $0.0-0.2$ & $1.56 \pm 0.51 \pm 0.12$ & $1.57 \pm 0.31 \pm 0.13$ & $1.22 \pm 0.68 \pm 0.12$ & $0.98 \pm 0.39 \pm 0.12$ & $1.20 \pm 0.38 \pm 0.12$ & $0.89 \pm 0.54 \pm 0.12$ \\
\hline $0.2-0.4$ & $1.65 \pm 0.51 \pm 0.12$ & $2.05 \pm 0.32 \pm 0.16$ & $1.60 \pm 0.73 \pm 0.16$ & $1.58 \pm 0.42 \pm 0.16$ & $1.98 \pm 0.40 \pm 0.16$ & $2.55 \pm 0.58 \pm 0.17$ \\
\hline $0.4-0.6$ & $2.49 \pm 0.61 \pm 0.19$ & $2.96 \pm 0.39 \pm 0.23$ & $1.51 \pm 0.84 \pm 0.21$ & $2.19 \pm 0.48 \pm 0.21$ & $1.81 \pm 0.46 \pm 0.21$ & $2.10 \pm 0.67 \pm 0.22$ \\
\hline $0.6-0.8$ & $3.91 \pm 1.00 \pm 0.29$ & $3.26 \pm 0.51 \pm 0.26$ & $3.45 \pm 1.17 \pm 0.28$ & $3.28 \pm 0.67 \pm 0.28$ & $2.05 \pm 0.65 \pm 0.28$ & $1.38 \pm 0.93 \pm 0.29$ \\
\hline $0.8-0.96$ & $6.77 \pm 1.80 \pm 0.50$ & $2.87 \pm 0.71 \pm 0.24$ & $-0.23 \pm 1.63 \pm 0.31$ & $3.34 \pm 0.94 \pm 0.31$ & $3.76 \pm 0.90 \pm 0.31$ & $2.63 \pm 1.29 \pm 0.31$ \\
\hline
\end{tabular}


TABLE XVIII: Differential cross section $d \sigma / d \cos \theta^{*}$ for the $e^{+} e^{-} \rightarrow e^{+} e^{-}, \mu^{+} \mu^{-}, \tau^{+} \tau^{-}$production measured by L3.

\begin{tabular}{|c|c|c|}
\hline \multirow[t]{2}{*}{$\cos \theta^{*}$ range } & \multicolumn{2}{|c|}{$d \sigma / d \cos \theta^{*}(\mathrm{pb})$} \\
\hline & $182.7 \mathrm{GeV}$ & $188.7 \mathrm{GeV}$ \\
\hline \multicolumn{3}{|c|}{$e^{+} e^{-}$} \\
\hline$-0.719--0.575$ & $0.404 \pm 0.157$ & $0.167 \pm 0.075$ \\
\hline$-0.575--0.432$ & $0.306 \pm 0.115 \pm 0.013$ & $0.300 \pm 0.065$ \\
\hline$-0.432--0.288$ & $0.532 \pm 0.127$ & $0.526 \pm 0.070$ \\
\hline$-0.288--0.144$ & $0.539 \pm 0.122$ & $0.524 \pm 0.067$ \\
\hline$-0.144-0.0$ & $0.930 \pm 0.153$ & $0.708 \pm 0.075$ \\
\hline $0.0-0.144$ & $0.930 \pm 0.153$ & $0.973 \pm 0.087$ \\
\hline $0.144-0.288$ & $1.638 \pm 0.199$ & $1.405 \pm 0.103$ \\
\hline $0.288-0.432$ & $2.779 \pm 0.259$ & $2.561 \pm 0.139$ \\
\hline $0.432-0.575$ & $5.025 \pm 0.347$ & $5.054 \pm 0.194$ \\
\hline $0.575-0.719$ & $12.39 \pm 0.54$ & $11.23 \pm 0.29$ \\
\hline \multicolumn{3}{|c|}{$\mu^{+} \mu^{-}$} \\
\hline$-0.9--0.7$ & $0.115 \pm 0.101$ & $0.008 \pm 0.037$ \\
\hline$-0.7--0.5$ & $0.057 \pm 0.044$ & $0.160 \pm 0.036$ \\
\hline$-0.5--0.3$ & $0.139 \pm 0.066$ & $0.138 \pm 0.034$ \\
\hline$-0.3--0.1$ & $0.080 \pm 0.049$ & $0.111 \pm 0.032$ \\
\hline$-0.1-0.1$ & $0.396 \pm 0.127$ & $0.193 \pm 0.050$ \\
\hline $0.1-0.3$ & $0.190 \pm 0.078$ & $0.393 \pm 0.056$ \\
\hline $0.3-0.5$ & $0.703 \pm 0.139$ & $0.383 \pm 0.056$ \\
\hline $0.5-0.7$ & $0.427 \pm 0.111$ & $0.521 \pm 0.066$ \\
\hline $0.7-0.9$ & $0.415 \pm 0.164$ & $0.569 \pm 0.079$ \\
\hline \multicolumn{3}{|c|}{$\tau^{+} \tau^{-}$} \\
\hline$-0.9--0.7$ & $0.103 \pm 0.098$ & $0.121 \pm 0.055$ \\
\hline$-0.7--0.5$ & $0.092 \pm 0.065$ & $0.161 \pm 0.043$ \\
\hline$-0.5--0.3$ & $0.209 \pm 0.087$ & $0.177 \pm 0.045$ \\
\hline$-0.3--0.1$ & $0.328 \pm 0.108$ & $0.145 \pm 0.042$ \\
\hline$-0.1-0.1$ & $0.272 \pm 0.101$ & $0.228 \pm 0.055$ \\
\hline $0.1-0.3$ & $0.255 \pm 0.099$ & $0.328 \pm 0.061$ \\
\hline $0.3-0.5$ & $0.375 \pm 0.119$ & $0.382 \pm 0.067$ \\
\hline $0.5-0.7$ & $0.529 \pm 0.143$ & $0.460 \pm 0.075$ \\
\hline $0.7-0.9$ & $1.079 \pm 0.299$ & $0.450 \pm 0.113$ \\
\hline
\end{tabular}


TABLE XIX: The $e^{+} e^{-}$and hadronic forward-backward asymmetry, as measured at LEP 2.

\begin{tabular}{|c|c|c|}
\hline \multirow[t]{2}{*}{$\sqrt{s}(\mathrm{GeV})$} & \multicolumn{2}{|c|}{$A_{\mathrm{FB}}$} \\
\hline & $e^{+} e^{-}$ & $q \bar{q}$ \\
\hline \multicolumn{3}{|c|}{ ALEPH } \\
\hline \multirow[t]{2}{*}{183} & - & $0.33 \pm 0.19(b \bar{b})$ \\
\hline & & $0.95 \pm 0.27_{-0.09}^{+0.11}(c \bar{c})$ \\
\hline 189 & - & $0.34 \pm 0.19 \pm 0.02(b \bar{b})$ \\
\hline
\end{tabular}

DELPHI

\begin{tabular}{|c|c|c|}
\hline 130.2 & $0.81 \pm 0.06 \pm 0.015$ & - \\
\hline 136.2 & $0.89 \pm 0.04 \pm 0.013$ & - \\
\hline 161.3 & $0.82 \pm 0.04 \pm 0.012$ & - \\
\hline 172.1 & $0.81 \pm 0.04 \pm 0.01$ & - \\
\hline 183 & $0.814 \pm 0.017$ & - \\
\hline 189 & $0.810 \pm 0.010$ & - \\
\hline 192 & $0.831 \pm 0.024$ & - \\
\hline 196 & $0.818 \pm 0.015$ & - \\
\hline 200 & $0.789 \pm 0.016$ & - \\
\hline 202 & $0.829 \pm 0.020$ & - \\
\hline \multicolumn{3}{|c|}{ L3 } \\
\hline 130 & $0.806 \pm 0.043 \pm 0.006$ & - \\
\hline 136.1 & $0.879 \pm 0.039 \pm 0.006$ & - \\
\hline 161.3 & $0.818 \pm 0.046 \pm 0.012$ & - \\
\hline 172.1 & $0.795 \pm 0.056 \pm 0.012$ & - \\
\hline 182.7 & $0.778 \pm 0.021 \pm 0.004$ & - \\
\hline 188.7 & $0.819 \pm 0.012 \pm 0.003$ & - \\
\hline \multicolumn{3}{|c|}{ OPAL } \\
\hline \multirow[t]{2}{*}{189} & \multirow[t]{2}{*}{-} & $0.43 \pm 0.15 \pm 0.08(b \bar{b})$ \\
\hline & & $0.57 \pm 0.18 \pm 0.09(c \bar{c})$ \\
\hline
\end{tabular}


TABLE XX: Dijet differential cross section $d \sigma / d M$ measured by CDF.

\begin{tabular}{|c|c|c|c|c|}
\hline $\begin{array}{l}\text { bin edge } \\
\left(\mathrm{GeV}^{2}\right)\end{array}$ & $\begin{array}{c}\text { average } M \\
\left(\mathrm{GeV}^{2}\right)\end{array}$ & $\begin{array}{c}d \sigma / d M \\
\left(\mathrm{pb} / \mathrm{GeV}^{2}\right)\end{array}$ & $\begin{array}{l}\text { statistical } \\
\text { uncertainty }\end{array}$ & $\begin{array}{l}\text { systematic } \\
\text { uncertainty }\end{array}$ \\
\hline 180 & 188 & $6.07 \times 10^{2}$ & $3.2 \%$ & ${ }_{-17}^{+20} \%$ \\
\hline 198 & 207 & $3.42 \times 10^{2}$ & $4.1 \%$ & ${ }_{-17}^{+19} \%$ \\
\hline 217 & 228 & $1.81 \times 10^{2}$ & $1.0 \%$ & ${ }_{-16}^{+19} \%$ \\
\hline 241 & 252 & $9.81 \times 10^{1}$ & $1.4 \%$ & ${ }_{-16}^{+19} \%$ \\
\hline 265 & 277 & $4.98 \times 10^{1}$ & $1.8 \%$ & $\begin{array}{l}+19 \% \\
-17 \%\end{array}$ \\
\hline 292 & 305 & $2.78 \times 10^{1}$ & $1.1 \%$ & ${ }_{-17}^{+19 \%}$ \\
\hline 321 & 335 & $1.43 \times 10^{1}$ & $1.4 \%$ & ${ }_{-17}^{+20} \%$ \\
\hline 353 & 368 & $7.41 \times 10^{0}$ & $1.9 \%$ & ${ }_{-18}^{+20} \%$ \\
\hline 388 & 405 & $3.83 \times 10^{0}$ & $0.9 \%$ & ${ }_{-18}^{+21} \%$ \\
\hline 427 & 446 & $1.89 \times 10^{0}$ & $1.2 \%$ & ${ }_{-19}^{+21} \%$ \\
\hline 470 & 491 & $9.07 \times 10^{-1}$ & $1.7 \%$ & ${ }_{-19}^{+22} \%$ \\
\hline 517 & 539 & $4.50 \times 10^{-1}$ & $2.3 \%$ & ${ }_{-20}^{+23} \%$ \\
\hline 568 & 592 & $1.90 \times 10^{-1}$ & $3.3 \%$ & ${ }_{-21}^{+25 \%}$ \\
\hline 625 & 652 & $7.42 \times 10^{-2}$ & $5.1 \%$ & $\begin{array}{l}+26 \% \\
-22\end{array}$ \\
\hline 688 & 716 & $2.92 \times 10^{-2}$ & $7.7 \%$ & ${ }_{-23}^{+28} \%$ \\
\hline 756 & 784 & $1.18 \times 10^{-2}$ & $11 \%$ & ${ }_{-25}^{+30} \%$ \\
\hline 832 & 865 & $3.57 \times 10^{-3}$ & $20 \%$ & $\begin{array}{l}+32 \% \\
-26\end{array}$ \\
\hline 915 & 968 & $9.03 \times 10^{-4}$ & $33 \%$ & ${ }_{-28}^{+34} \%$ \\
\hline
\end{tabular}


TABLE XXI: Dijet invariant mass distribution measured by D $\varnothing$ for $\left|\eta_{\text {jet }}\right|<1$.

\begin{tabular}{cccc}
\hline \hline$M_{j j}$ mass bin $(\mathrm{GeV})$ & & \multicolumn{2}{c}{$d^{3} / d M_{j j} d \eta_{1} d \eta_{2}(\mathrm{nb} / \mathrm{GeV})$} \\
& \pm stat. error & syst. low $(\%)$ & syst. high $(\%)$ \\
\hline $200-220$ & $(3.78 \pm 0.12) \cdot 10^{-2}$ & -11.4 & +11.8 \\
$220-240$ & $(2.10 \pm 0.09) \cdot 10^{-2}$ & -11.3 & +11.6 \\
$240-270$ & $(1.16 \pm 0.06) \cdot 10^{-2}$ & -11.5 & +11.7 \\
$270-300$ & $(6.18 \pm 0.11) \cdot 10^{-3}$ & -11.5 & +12.0 \\
$300-320$ & $(3.55 \pm 0.11) \cdot 10^{-3}$ & -11.5 & +12.1 \\
$320-350$ & $(2.12 \pm 0.07) \cdot 10^{-3}$ & -11.9 & +12.3 \\
$350-390$ & $(1.18 \pm 0.01) \cdot 10^{-3}$ & -11.1 & +11.6 \\
$390-430$ & $(5.84 \pm 0.09) \cdot 10^{-4}$ & -11.5 & +12.2 \\
$430-470$ & $(2.89 \pm 0.06) \cdot 10^{-4}$ & -11.9 & +12.9 \\
$470-510$ & $(1.64 \pm 0.05) \cdot 10^{-4}$ & -12.4 & +13.5 \\
$510-550$ & $(8.74 \pm 0.34) \cdot 10^{-5}$ & -12.8 & +14.3 \\
$550-600$ & $(4.49 \pm 0.17) \cdot 10^{-5}$ & -13.5 & +15.3 \\
$600-700$ & $(1.73 \pm 0.07) \cdot 10^{-5}$ & -14.9 & +17.2 \\
$700-800$ & $(4.58 \pm 0.38) \cdot 10^{-6}$ & -17.6 & +20.8 \\
$800-1400$ & $(2.39 \pm 0.35) \cdot 10^{-7}$ & -23.2 & +28.9 \\
\hline \hline
\end{tabular}


TABLE XXII: Dijet angular distribution in various dijet invariant mass bins measured by (a) DØ and (b) CDF. DØ defines $R_{\chi} \equiv \frac{N(\chi<4)}{N\left(4<\chi<\chi_{\max }\right)}$ while CDF defines $R_{\chi} \equiv \frac{N(\chi<2.5)}{N(2.5<\chi<5)}$. The covariance matrix is given by $V_{i i}=\sigma_{i}^{2}$ (stat) $+\sigma_{i}^{2}$ (syst.) and $V_{i j}=\sigma_{i}$ (syst.) $\sigma_{j}$ (syst.).

(a) $\mathrm{D} \varnothing$

\begin{tabular}{|c|c|c|c|}
\hline$M_{j j}$ range $(\mathrm{GeV})$ & $R_{\chi}$ & Stat. error & Syst. error \\
\hline $260-425$ & 0.191 & 0.0077 & 0.015 \\
\hline $425-475$ & 0.202 & 0.0136 & 0.010 \\
\hline $475-635$ & 0.342 & 0.0085 & 0.018 \\
\hline$>635$ & 0.506 & 0.0324 & 0.028 \\
\hline \multicolumn{4}{|c|}{ (b) $\mathrm{CDF}$} \\
\hline $241-300$ & 0.678 & 0.012 & 0.018 \\
\hline $300-400$ & 0.695 & 0.010 & 0.025 \\
\hline $400-517$ & 0.703 & 0.009 & 0.033 \\
\hline $517-625$ & 0.738 & 0.023 & 0.054 \\
\hline$>625$ & 0.732 & 0.046 & 0.103 \\
\hline
\end{tabular}

